\title{
LOCAL VANISHING PROPERTIES OF SOLUTIONS OF ELLIPTIC AND PARABOLIC QUASILINEAR EQUATIONS
}

\author{
BY
}

\author{
J. ILDEFONSO DIAZ AND LAURENT VERON
}

\begin{abstract}
We use a local energy method to study the vanishing property of the weak solutions of the elliptic equation $-\operatorname{div} A(x, u, D u)+B(x, u, D u)=0$ and of the parabolic equation $\partial \psi(u) / \partial t-\operatorname{div} A(t, x, u, D u)+B(t, x, u, D u)=0$. The results are obtained without any assumption of monotonicity on $A, B, A$ and $B$.
\end{abstract}

1. Introduction. In this paper we study some local vanishing properties of weak solutions of elliptic and parabolic quasilinear equations of the following form:

$$
-\operatorname{div} A(x, u, D u)+B(x, u, D u)+C(x, u)=0,
$$

and

$$
\frac{\partial}{\partial t} \psi(u)-\operatorname{div} \mathcal{A}(t, x, u, D u)+B(t, x, u, D u)+C(t, x, u)=0,
$$

where $A$ (resp. $A$ ) is a vector valued function defined in $\Omega \times \mathbf{R} \times \mathbf{R}^{N}$ (resp. $\mathbf{R}^{+} \times \Omega \times$ $\left.\mathbf{R} \times \mathbf{R}^{N}\right), B$ (resp. B) is a real function defined in $\Omega \times \mathbf{R} \times \mathbf{R}^{N}\left(\right.$ resp. $\left.\mathbf{R}^{+} \times \Omega \times \mathbf{R} \times \mathbf{R}^{N}\right)$ and $C$ (resp. C) a real valued function defined in $\Omega \times \mathbf{R}$ (resp. $\mathbf{R}^{+} \times \Omega \times \mathbf{R}$ ) and where $\psi$ is a continuous nondecreasing function vanishing at 0 . The functions $A$, $B, C, A, B, C$ and $\psi$ are required to satisfy the following structural assumptions for some constants $C_{1}, \ldots, C_{4}, M_{1}, \ldots, M_{4}$ and $c$ and some exponents $q, \alpha, \beta, \sigma$ and $m$ which will be made precise later.

$$
\begin{gathered}
|A(x, r, p)| \leq C_{1}|p|^{q} \quad \forall(x, r, p) \in \Omega \times \mathbf{R} \times \mathbf{R}^{N}, \\
A(x, r, p) \cdot p \geq C_{2}|p|^{q+1} \quad \forall(x, r, p) \in \Omega \times \mathbf{R} \times \mathbf{R}^{N}, \\
|B(x, r, p)| \leq C_{3}|r|^{\alpha}|p|^{\beta} \quad \forall(x, r, p) \in \Omega \times \mathbf{R} \times \mathbf{R}^{N}, \\
C(x, r) r \geq C_{4}|r|^{\sigma+1} \quad \forall(x, r) \in \Omega \times \mathbf{R}, \\
|\mathcal{A}(t, x, r, p)| \leq M_{1}|p|^{q} \quad \forall(t, x, r, p) \in \mathbf{R}^{+} \times \Omega \times \mathbf{R} \times \mathbf{R}^{N}, \\
\mathcal{A}(t, x, r, p) \cdot p \geq M_{2}|p|^{q+1} \quad \forall(t, x, r, p) \in \mathbf{R}^{+} \times \Omega \times \mathbf{R} \times \mathbf{R}^{N}, \\
|B(t, x, r, p)| \leq M_{3}|r|^{\alpha}|p|^{\beta} \quad \forall(t, x, r, p) \in \mathbf{R}^{+} \times \Omega \times \mathbf{R} \times \mathbf{R}^{N}, \\
C(t, x, r) r \geq M_{4}|r|^{\sigma+1} \quad \forall(t, x, r) \in \mathbf{R}^{+} \times \Omega \times \mathbf{R}, \\
f(r)=r \psi(r)-j(r) \geq c|r|^{(m+1) / m} \quad \forall r \in \mathbf{R},
\end{gathered}
$$

Received by the editors September 28, 1984.

1980 Mathematics Subject Classification. Primary 35B45, 35J60, 35K60.

Key words and phrases. Elliptic equations, parabolic equations, weak solutions, local energy estimates, differential inequalities, trace-interpolation estimates, free boundary, propagation phenomena. 
where $j(r)=\int_{0}^{r} \psi(s) d s$ satisfies the $\Delta_{2}$ condition. An important particular case of $(\mathrm{EE})$ is the equation

$$
-\operatorname{div}\left(|D u|^{q-1} D u\right)+|u|^{\sigma-1} u=0
$$

which appears in the study of a stationary isothermical single reaction $[\mathbf{4}]$ or in the non-Newtonian stationary fluids theory $[\mathbf{2 9}](q>0, \sigma=1)$. As for (PE), an interesting particularization is

$$
\frac{\partial v}{\partial t}-\operatorname{div}\left(\left|D\left(v|v|^{m-1}\right)\right|^{q-1} D\left(v|v|^{m-1}\right)\right)+|v|^{\tilde{\sigma}-1} v=0
$$

which appears in filtration with absorption of gases in porous media $(q=1)[20]$, spatial diffusion of a biological population [18] and in the study of nonstationary non-Newtonian fluids $(m=\tilde{\sigma}=1)$ [29]. It must be noticed that (1.2) is linked to (PE) in setting $\sigma=\tilde{\sigma} / m$ and $u=v|v|^{m-1}$.

Our paper deals with the following vanishing properties:

(A) if $u$ is a weak solution of (EE) in $B_{\rho_{0}}\left(x_{0}\right)=\left\{x \in \Omega:\left|x-x_{0}\right|<\rho_{0}\right\}$, then there exists $\rho_{1}, 0 \leq \rho_{1}<\rho_{0}$, such that $u(x)=0 \forall x \in B_{\rho_{1}}\left(x_{0}\right)$;

(B) if $u$ is a weak solution of (PE) in $\mathbf{R}^{+} \times B_{\rho_{0}}\left(x_{0}\right)$ and $u(0, x)=0 \forall x \in$ $B_{\rho_{0}}\left(x_{0}\right)$, then for any $t<t_{0}$ there exists $\rho(t), 0 \leq \rho(t)<\rho_{0}$, such that $u(t, x)=0$ $\forall x \in B_{\rho(t)}\left(x_{0}\right)$;

(C) if $u$ is a weak solution of $(\mathrm{PE})$ in $\mathbf{R}^{+} \times B_{\rho_{0}}\left(x_{0}\right)$ and $u(0, x)=0 \forall x \in B_{\rho_{0}}\left(x_{0}\right)$, then there exists $\rho_{1}, 0 \leq \rho_{1}<\rho_{0}$, such that $u(t, x)=0 \forall(t, x) \in \mathbf{R}^{+} \times B_{\rho_{1}}\left(x_{0}\right)$.

If, for the sake of simplicity, we assume $C_{3}=M_{3}=0$, our main results can be summarized in the following way:

(I) under hypotheses (E1)-(E4) property (A) holds if we suppose $C_{2}>0, C_{4}>0$ and $0 \leq \sigma<q$;

(II) under hypotheses (P1)-(P5) property (B) holds if we suppose $M_{2}>0, M_{4} \geq$ $0, \sigma \geq 0$ and $m q>1$

(III) under hypotheses (P1)-(P5) property (C) holds if we suppose $M_{2}>0$, $M_{4}>0, \sigma \geq 0, m>0, q>\max (\sigma, 1 / m)$ and if the "energy" of $u$ in $\mathbf{R}^{+} \times B_{\rho_{0}}\left(x_{0}\right)$ is finite.

As a consequence of those three local vanishing estimates we have the following three global results.

TheOREM I. Assume $C_{2}>0, C_{4}>0,0 \leq \sigma<q$ and $u \in L^{\sigma+1}\left(\mathbf{R}^{N}\right) \cap$ $W^{1, q+1}\left(\mathbf{R}^{N}\right)$ is any weak solution of

$$
-\operatorname{div} A(x, u, D u)+C(x, u)=f(x)
$$

in $\mathbf{R}^{N}$, where $f \in L^{(\sigma+1) / \sigma}\left(\mathbf{R}^{N}\right)$. If $f$ has its support in $B_{\rho_{0}}(0)$, then there exists $\rho_{1}>\rho_{0}$ depending on $\|f\|_{L^{(\sigma+1) / \sigma}\left(\mathbf{R}^{N}\right)}$ and the structural constants $C_{1}, C_{2}, C_{4}, q$, $\sigma$ and $N$ such that $\operatorname{supp} u(\cdot) \subset B_{\rho_{1}}(0)$.

THEOREM II. Assume $M_{2}>0, M_{4} \geq 0, c>0, m>0, m q>1$ and $u$ is a weak solution in $\mathbf{R}^{+} \times \mathbf{R}^{N}$ of

$$
\frac{\partial}{\partial t} \psi(u)-\operatorname{div} \mathcal{A}(t, x, u, D u)+C(t, x, u)=0
$$


such that $j(u) \in C^{0}\left(\mathbf{R}^{+} ; L_{\text {loc }}^{1}\left(\mathbf{R}^{N}\right)\right)$. Assume also that for any $\rho>0$ and $t>0$ there exists a constant $K=K(t, \rho)$ such that for any $y \in \mathbf{R}^{N}$

$$
\underset{\tau \leq t}{\sup \operatorname{ess}} \int_{B_{\rho}(y)} f(u(\tau, x)) d x+\int_{0}^{t} \int_{B_{\rho}(y)} A(\tau, x, u, D u) \cdot D u d x d \tau \leq K .
$$

If the initial data $u_{0}$ of $u$ vanishes outside $B_{R}(0)$, then there exists a nondecreasing function defined on $\mathbf{R}^{+}$such that $R(0)=R$ and $\operatorname{supp} u(t, \cdot) \subset B_{R(t)}(0)$ for any $t \geq 0$.

Theorem III. Assume $M_{2}>0, M_{4}>0, \sigma \geq 0, m>0, c>0, \max (\sigma, 1 / m)<$ $q$ and $u$ is any weak solution of (1.4) in $\mathbf{R}^{+} \times \mathbf{R}^{N}$ such that

$$
j(u) \in C^{0}\left(\mathbf{R}^{+} ; L_{\text {loc }}^{1}\left(\mathbf{R}^{N}\right)\right) .
$$

Assume also that $u$ satisfies the same condition (1.5) as in Theorem II. If the initial data $u_{0}$ of $u$ vanishes outside $B_{R}(0)$, then there exists $R_{1}>R$ such that $\operatorname{supp} u(t, \cdot) \subset B_{R_{1}}(0)$ for any $t \geq 0$.

More general results involving $C_{3}, M_{3}, \alpha$ and $\beta$ will be given in the sequel. The phenomena described in (A), (B) and (C) are already known for solutions of (1.1) and $(1.2)([11,13,14,16,19]$, etc.) but they have always been obtained on the basis of comparison principles where the monotonicity of the different operators is crucial. On the contrary our method is just an energy method and no assumption of monotonicity on $A, B, C, A, B$ and $C$ is needed. Moreover it is a unified method and the proofs of I, II and III are parallel. Roughly speaking, the idea is to multiply the equation (EE) or (PE) by $u$, to integrate in some ball of radius $\rho, B_{\rho}\left(x_{0}\right)$, and to use Green's formula. We then obtain a first order differential inequality involving the energy $E(\rho)$ of $u$ concentrated in $B_{\rho}\left(x_{0}\right)$ of the type

$$
K \rho^{\alpha_{0}} \frac{d}{d \rho} E(\rho) \geq(E(\rho))^{\alpha_{1}} .
$$

In case (B), $K=K(t)$ is a power of $t$. Integrating (1.6) we obtained estimates for $\rho_{1}=\rho_{1}\left(\rho_{0}, E\left(\rho_{0}\right)\right)$ such that $E\left(\rho_{1}\right)=0$ and then

$$
u(\cdot)=0 \quad \text { a.e. in } B_{\rho_{1}}\left(x_{0}\right)
$$

( $\rho_{1}$ depends also on $t$ in case (B)). In cases (B) and (C) we also assume $u(0, \cdot)=0$ a.e. in $B_{\rho_{0}}\left(x_{0}\right)$. A key-stone tool for such a program is an interpolation-trace result which will be proved in $\S 4$.

This energy method was first introduced by Antoncev [3] in a pioneering but very formal work where he essentially obtained a result of type II in the framework of Lions' existence results [28] for solutions of (PE). We give here a rigourous proof of his result in a more general situation.

It must also be noticed that our results hold in some cases of variational inequalities (take $\sigma=0$ ).

ACKNOWLEDGEMENTS. This paper was partially written while the second author was visiting the Universidad Complutense in the framework of the Scientific Cooperation between France and Spain. 
2. The elliptic equation. In this section $\Omega$ is an open subset of $\mathbf{R}^{N}, N \geq \mathbf{1}$, $A, B$ and $C$ are Carathéodory vector valued (for $A$ ) or real valued (for $B$ and $C$ ) functions defined in $\Omega \times \mathbf{R} \times \mathbf{R}^{N}$ (for $A$ and $B$ ) or in $\Omega \times \mathbf{R}$ (for $C$ ) satisfying (E1), (E2), (E3) and (E4).

DEFINITION 2.1. A locally integrable function $u$ defined in $\Omega$ is a weak solution of (EE) if

(i) $D u \in L_{\text {loc }}^{q+1}(\Omega), q>0$,

(ii) $B(\cdot, u, D u) \in L_{\text {loc }}^{1}(\Omega)$,

(iii) $C(\cdot, u) \in L_{\text {loc }}^{1}(\Omega)$,

and for any $\varphi \in C_{0}^{\infty}(\Omega)$ the following equality holds:

$$
\int_{\Omega}\{A(x, u, D u) \cdot D \varphi+B(x, u, D u) \varphi+C(x, u) \varphi\} d x=0 .
$$

We set $B_{\rho}\left(x_{0}\right)=\left\{x:\left|x-x_{0}\right|<\rho\right\}$ and $S_{\rho}\left(x_{0}\right)=\partial B_{\rho}\left(x_{0}\right)$. Our main result is the following

THEOREM 2.1. Suppose $C_{2}>0, C_{4}>0,0 \leq \sigma<q, 0 \leq \beta \leq q+1, \alpha=$ $\sigma-\beta(\sigma+1) /(q+1)$ and $C_{3}<C_{4}\left(\right.$ resp. $\left.C_{3}<C_{2}\right)$ if $\beta=0($ resp. $\beta=q+1)$ or

$$
C_{3}<\left(C_{4} \frac{q+1}{q+1-\beta}\right)^{(q+1-\beta) /(q+1)}\left(C_{2} \frac{q+1}{\beta}\right)^{\beta /(q+1)}
$$

if $0<\beta<q+1$. If $u$ is a weak solution of (EE) in $\Omega, x_{0} \in \Omega$ and $\rho_{0}$ is such that $0<\rho_{0}<\operatorname{dist}\left(x_{0}, \partial \Omega\right)$, then $u(x)=0$ a.e. in $B_{\rho_{1}}\left(x_{0}\right)$, where

$\rho_{1}^{\nu}=\rho_{0}^{\nu}-C \min _{(\sigma+1) /(q+1)<\tau \leq 1}\left\{\frac{E^{\gamma}\left(\rho_{0}\right)}{\tau(q+1)-\sigma-1} \max \left(1, \rho_{0}^{\nu-1}\right) \max \left(b^{\mu}\left(\rho_{0}\right), b^{\eta}\left(\rho_{0}\right)\right)\right\}$, where $C=C\left(C_{1}, C_{2}, C_{3}, C_{4}, N, q, \sigma, \beta\right)$ and

$$
\begin{gathered}
E(\rho)=\int_{B_{\rho}\left(x_{0}\right)} A(x, u, D u) \cdot D u d x, \quad b(\rho)=\int_{B_{\rho}\left(x_{0}\right)}|u|^{\sigma+1} d x, \\
\kappa=N(q-\sigma)+(\sigma+1)(q+1), \quad \eta=\frac{q-\sigma}{q(\sigma+1)}-\frac{\sigma+1-\tau(q+1)}{\kappa}, \\
\gamma=\frac{\tau(q+1)-\sigma-1}{\kappa}, \quad \mu=\frac{(1-\tau)(q+1)}{\kappa}, \quad \nu=\frac{\kappa}{q(\sigma+1)} .
\end{gathered}
$$

REMARK 2.1. If $\rho_{1} \leq 0$, then $B_{\rho_{1}}\left(x_{0}\right)$ is empty and we have no information on the vanishing set of $u$. But if the total energy of $u$ in $B_{\rho_{0}}\left(x_{0}\right)$ defined by $E\left(\rho_{0}\right)+b\left(\rho_{0}\right)$ is not too large, then $\rho_{1}>0$ and there truly exists a vanishing set of $u$ in $B_{\rho_{0}}\left(x_{0}\right)$. The exact value of $\rho_{1}$ is not easy to compute through (2.3), moreover if $\min \left(b\left(\rho_{0}\right), E\left(\rho_{0}\right)\right)=0$, then $\rho_{0}=\rho_{1}$, which was obvious from the hypotheses.

LEMMA 2.1. Under the hypotheses of Theorem 2.1, $A(\cdot, u, D u) \cdot D u,|u|^{\sigma+1}$, $|A(\cdot, u, D u)| u$ and $B(\cdot, u, D u) u$ belong to $L^{1}\left(B_{\rho_{0}}\left(x_{0}\right)\right)$ and for almost every $\rho \in$ $\left(0, \rho_{0}\right)$ we have

$$
\begin{gathered}
\int_{B_{\rho}\left(x_{0}\right)} A(x, u, D u) \cdot D u d x+C_{4} \int_{B_{\rho}\left(x_{0}\right)}|u|^{\sigma+1} d x+\int_{B_{\rho}\left(x_{0}\right)} B(x, u, D u) u d x \\
\leq \int_{S_{\rho}\left(x_{0}\right)} A(x, u, D u) \cdot \vec{\nu} u d s,
\end{gathered}
$$

where $\vec{\nu}=\vec{\nu}(x)$ is the outward normal vector at $x \in S_{\rho}\left(x_{0}\right)$. 
ProOF. From the definition we deduce with standard Poincaré and Sobolev inequalities arguments that $u \in L^{r}\left(B_{\rho_{0}}\left(x_{0}\right)\right)$ with $1 / r=1 /(q+1)-1 / N$ if $q+1<N$ or $r<+\infty$ if $q+1 \geq N$. Hence $u \in L^{\sigma+1}\left(B_{\rho_{0}}\left(x_{0}\right)\right)$. From Hölder's inequality and (E3) $u B(x, u, D u)$ is integrable in $B_{\rho_{0}}\left(x_{0}\right)$ and it is the same with $|A(x, u, D u)| u$ so $\int_{S_{\rho}\left(x_{0}\right)} A(x, u, D u) \cdot \vec{\nu} u d s$ exists for almost all $\rho$ in $\left(0, \rho_{0}\right)$.

We now define for $m \in \mathbf{N}, T_{m}(u)=\operatorname{sign}(u) \min (m,|u|)$ and for $n \in \mathbf{N}$ and $\rho \in\left(0, \rho_{0}\right)$, we consider the sequence of functions $\psi_{n}:\left[0, \rho_{0}\right] \mapsto \mathbf{R}^{+}$such that

$$
\psi_{n}(r)= \begin{cases}1 & \text { if } r \in[0, \rho-1 / n] \\ 0 & \text { if } r \in\left[\rho, \rho_{0}\right], \\ -n(\rho-r) & \text { if } r \in[\rho-1 / n, \rho]\end{cases}
$$

From a result of Stampacchia $[\mathbf{3 3}], \varphi_{n, m}(x)=T_{m}(u(x)) \psi_{n}\left(\left|x-x_{0}\right|\right)$ belongs to $W_{0}^{1, q+1}\left(B_{\rho_{0}}\left(x_{0}\right)\right)$ so it is an admissible test function and we have

$$
\int_{B_{\rho_{0}}\left(x_{0}\right)}\left\{A(x, u, D u) \cdot D \varphi_{n, m}+B(x, u, D u) \varphi_{n, m}+C(x, u) \varphi_{n, m}\right\} d x=0
$$

But

$$
\begin{aligned}
\int_{B_{\rho_{0}}\left(x_{0}\right)} A(x, u, D u) \cdot D \varphi_{n, m} d x \\
\quad=\int_{B_{\rho}\left(x_{0}\right)}\left\{\psi_{n} A(x, u, D u) \cdot D T_{m}(u)+T_{m}(u) A(x, u, D u) \cdot D \psi_{n}\right\} d x
\end{aligned}
$$

We deduce from Lebesgue's theorem as $m$ goes to infinity that

$$
\begin{gathered}
\int_{B_{\rho_{0}}\left(x_{0}\right)} \psi_{n}\left\{A(x, u, D u) \cdot D u+B(x, u, D u) u+C_{4}|u|^{\sigma+1}\right\} d x \\
\leq-\int_{B_{\rho_{0}}\left(x_{0}\right)} u A(x, u, D u) \cdot D \psi_{n} d x
\end{gathered}
$$

But

$$
-\int_{B_{\rho_{0}}\left(x_{0}\right)} u A(x, u, D u) \cdot D \psi_{n} d x=n \int_{\rho-1 / n<\left|x-x_{0}\right|<\rho} u A(x, u, D u) \cdot \frac{x-x_{0}}{\left|x-x_{0}\right|} d x .
$$

Using spherical coordinates $(r, \omega)$ with center $x_{0}$ we have

$$
\begin{aligned}
n \int_{\rho-1 / n<\left|x-x_{0}\right|<\rho} u A(x, u, D u) \cdot \frac{x-x_{0}}{\left|x-x_{0}\right|} d x \\
=n \int_{\rho-1 / n}^{\rho} \int_{S^{N-1}} u A(x, u, D u) \cdot \vec{\nu} r^{N-1} d \omega d r
\end{aligned}
$$

where $x=r \omega$. From Lebesgue's differentiation theorem and the fact that $r \mapsto$ $\int_{S^{N-1}} u A(r \omega, u, D u) \cdot \vec{\nu} r^{N-1} d \omega \in L^{1}\left(0, \rho_{0}\right)$, we deduce that for almost all $\rho \in$ $\left(0, \rho_{0}\right)$,

$$
\lim _{n \rightarrow \infty} n \int_{\rho-1 / n}^{\rho} \int_{S^{N-1}} u A(r \omega, u, D u) \cdot \vec{\nu} r^{N-1} d \omega=\int_{S_{\rho}\left(x_{0}\right)} u A(x, u, D u) \cdot \vec{\nu} d s
$$

Going to the limit $(n \rightarrow \infty)$ in (2.10) we deduce (2.7).

The keystone of the proof of Theorem 2.1 is the following interpolation-trace result whose proof will be given in $\S 4$. 
LEMmA 2.2. Suppose $G$ is a bounded open subset of $R^{N}, N \geq 1$, with a $C^{1}$ boundary $\partial G$ and $0 \leq \sigma \leq q<\infty$. Then there exists a constant $C$ depending on $\sigma$, $q$ and $G$ such that for any $v \in W^{1, q+1}(G)$ we have

$$
\|v\|_{L^{q+1}(\partial G)} \leq C\left(\|D v\|_{L^{q+1}(G)}+\|v\|_{L^{\sigma+1}(G)}\right)^{\theta}\|v\|_{L^{\sigma+1}(G)}^{1-\theta},
$$

where $\theta=(N(q-\sigma)+\sigma+1) / \kappa$.

As a consequence we have

COROLlaRY 2.1. If in Lemma 2.2 we suppose that $G=B_{\rho}\left(x_{0}\right), \rho>0$, then for any $u \in W^{1, q+1}\left(B_{\rho}\left(x_{0}\right)\right)$ we have

$$
\|u\|_{L^{q+1}\left(S_{\rho}\left(x_{0}\right)\right)} \leq C\left(\|D u\|_{L^{q+1}\left(B_{\rho}\left(x_{0}\right)\right)}+\rho^{\delta}\|u\|_{L^{\sigma+1}\left(B_{\rho}\left(x_{0}\right)\right)}\right)^{\theta}\|u\|_{L^{\sigma+1}\left(3_{\rho}\left(x_{0}\right)\right)}^{1-\theta}
$$

where $\delta=\kappa /(q+1)(\sigma+1)$ and $C=C(N, \sigma, q)$.

PROOF. For the sake of simplicity we suppose $x_{0}=0$ and we perform the following change of variable: $x=\rho y, x \in B_{\rho}(0), y \in B_{1}(0)$. If $u \in W^{1, q+1}\left(B_{\rho}(0)\right)$, the function $v$ defined by $v(y)=u(x)$ belongs to $W^{1, q+1}\left(B_{1}(0)\right)$ and from $(2.12)$ we have

$$
\|v\|_{L^{q+1}\left(S_{1}(0)\right)} \leq C\left(\|D v\|_{L^{q+1}\left(B_{1}(0)\right)}+\|v\|_{L^{\sigma+1}\left(B_{1}(0)\right)}\right)^{\theta}\|v\|_{L^{\sigma+1}\left(B_{1}(0)\right)}^{1-\theta} .
$$

But $D v(y)=\rho D u(x)$,

$$
\begin{aligned}
\|v\|_{L^{\sigma+1}\left(B_{1}(0)\right)} & =\rho^{-N /(\sigma+1)}\|u\|_{L^{\sigma+1}\left(B_{\rho}(0)\right)}, \\
\|D v\|_{L^{q+1}\left(B_{1}(0)\right)} & =\rho^{1-N /(q+1)}\|D u\|_{L^{q+1}\left(B_{\rho}(0)\right)},
\end{aligned}
$$

and

$$
\|v\|_{L^{q+1}\left(S_{1}(0)\right)}=\rho^{-(N-1) /(q+1)}\|u\|_{L^{q+1}\left(S_{\rho}(0)\right)} .
$$

As

and

$$
1-\frac{N}{q+1}+\frac{N-1}{\theta(q+1)}-\frac{1-\theta}{\theta} \frac{N}{\sigma+1}=0
$$

$$
-\frac{N}{\sigma+1}+\frac{N-1}{\theta(q+1)}-\frac{1-\theta}{\theta} \frac{N}{\sigma+1}=-\frac{N(q-\sigma)+(\sigma+1)(q+1)}{(q+1)(\sigma+1)}
$$

we get (2.13).

PROOF OF THEOREM 2.1.

First step. There exists a constant $C_{5}=C_{5}\left(C_{2}, C_{4}, q, \sigma, \beta\right), C_{5}>0$, such that

$$
E(\rho)+C_{4} b(\rho)+\int_{B_{\rho}\left(x_{0}\right)} B(x, u, D u) u d x \geq C_{5}(E(\rho)+b(\rho)) .
$$

When $\beta=0$ or $\beta=q+1$ this is clear; when $0<\beta<q+1$ this can be seen as follows: from (E3) we have

$$
\left|\int_{B_{\rho}\left(x_{0}\right)} B(x, u, D u) u d x\right| \leq C_{3} \int_{B_{\rho}\left(x_{0}\right)}|u|^{\alpha+1}|D u|^{\beta} d x .
$$

Using Young's inequality, we have for any $\varepsilon>0$ and $\tau>1$

$$
|u|^{\alpha+1}|D u|^{\beta} \leq \frac{\varepsilon}{\tau}|u|^{\tau(\alpha+1)}+\frac{(\tau-1)}{\tau} \varepsilon^{-1 /(\tau-1)}|D u|^{\beta \tau /(\tau-1)} .
$$


If we choose $\tau=(\sigma+1) /(\alpha+1)$, then $\beta \tau /(\tau-1)=q+1$ and

$$
|u|^{\alpha+1}|D u|^{\beta} \leq \varepsilon \frac{q+1-\beta}{q+1}|u|^{\sigma+1}+\frac{\beta}{q+1} \varepsilon^{-(q+1-\beta) / \beta}|D u|^{q+1},
$$

which implies

$$
\left|\int_{B_{\rho}\left(x_{0}\right)} B(x, u, D u) u d x\right| \leq \varepsilon C_{3} \frac{q+1-\beta}{q+1} b(\rho)+\frac{\beta C_{3}}{C_{2}(q+1)} \varepsilon^{-(q+1-\beta) / \beta} E(\rho) .
$$

As $C_{3}$ satisfies (2.2) it is possible to find $\varepsilon>0$ depending on $q, \beta, C_{2}, C_{4}$ such that

$$
\varepsilon C_{3} \frac{q+1-\beta}{q+1}<C_{4} \text { and } \frac{\beta C_{3}}{C_{2}(q+1)} \varepsilon^{-(q+1-\beta) / \beta}<1 .
$$

If we set

$$
C_{5}=\min \left\{C_{4}-\varepsilon C_{3} \frac{q+1-\beta}{q+1}, 1-\frac{\beta C_{3}}{C_{2}(q+1)} \varepsilon^{-(q+1-\beta) / \beta}\right\}
$$

we get (2.15).

End of proof. From (2.7) and (2.15) we have

$$
C_{5}(E(\rho)+b(\rho)) \leq \int_{S_{\rho}\left(x_{0}\right)} A(x, u, D u) \cdot \vec{\nu} u d s
$$

and

$$
\begin{aligned}
& \int_{S_{\rho}\left(x_{0}\right)} A(x, u, D u) \cdot \vec{\nu} u d s \\
& \quad \leq C_{1}\left(\int_{S_{\rho}\left(x_{0}\right)}|D u|^{q+1} d s\right)^{q /(q+1)}\left(\int_{S_{\rho}\left(x_{0}\right)}|u|^{q+1} d s\right)^{1 /(q+1)} .
\end{aligned}
$$

In spherical coordinates $(\omega, r)$ with center $x_{0}$ we have

$$
E(\rho)=\int_{0}^{\rho} \int_{S^{N-1}} A(r \omega, u, D u) \cdot D u r^{N-1} d \omega d r
$$

hence $E$ is almost everywhere differentiable and $d E(\rho) / d \rho=\int_{S^{N-1}} A(\rho \omega, u, D u)$. $D u \rho^{N-1} d \omega$ and from (E2) $d E(\rho) / d \rho \geq C_{2} \int_{S_{\rho}\left(x_{0}\right)}|D u|^{q+1} d s$. So we get (using

$$
E(\rho)+b(\rho) \leq K\left(\frac{d E}{d \rho}\right)^{q /(q+1)}\left(E(\rho)^{1 /(q+1)}+\rho^{\delta} b(\rho)^{1 /(q+1)}\right)^{\theta} b(\rho)^{(1-\theta) /(\sigma+1)}
$$

where $K=K\left(C_{1}, C_{2}, C_{5}, N, \sigma, q, \beta\right)$. Moreover for $0 \leq \tau \leq 1$, we have

$$
\begin{aligned}
E(\rho)^{1 /(q+1)} b(\rho)^{(1-\theta) / \theta(\sigma+1)}+\rho^{\delta} b(\rho)^{1 / \theta(\sigma+1)} & \\
= & E(\rho)^{1 /(q+1)} b(\rho)^{\tau(1-\theta) / \theta(\sigma+1)} b(\rho)^{(1-\tau)(1-\theta) / \theta(\sigma+1)}+\cdots \\
& +\rho^{\delta} b(\rho)^{1 /(q+1)+\tau(1-\theta) / \theta(\sigma+1)} b(\rho)^{1 / \theta(\sigma+1)-1 /(q+1)-\tau(1-\theta) / \theta(\sigma+1)} .
\end{aligned}
$$

If we set

$$
K_{0}=\max \left(b\left(\rho_{0}\right)^{(1-\tau)(1-\theta) /(\sigma+1)}, b\left(\rho_{0}\right)^{(1-\tau(1-\theta)) /(\sigma+1)-\theta /(q+1)}\right)
$$


we get from Young's inequality

$$
\begin{aligned}
& E(\rho)^{1 /(q+1)} b(\rho)^{(1-\theta) / \theta(\sigma+1)}+\rho^{\delta} b(\rho)^{1 / \theta(\sigma+1)} \\
& \quad \leq 2 \rho^{\delta} K_{0}^{1 / \theta} \max \left(1, \rho_{0}^{-\delta}\right)(E(\rho)+b(\rho))^{1 /(q+1)+\tau(1-\theta) / \theta(\sigma+1)} .
\end{aligned}
$$

Hence we deduce

$$
(E(\rho)+b(\rho))^{1-\theta /(q+1)-\tau(1-\theta) /(\sigma+1)} \leq 2 K \rho^{\delta \theta} K_{0} \max \left(1, \rho_{0}^{-\delta \theta}\right)\left(\frac{d E}{d \rho}\right)^{q /(q+1)} .
$$

Let $K_{1}=\left(2 K K_{0} \max \left(1, \rho_{0}^{-\delta \theta}\right)\right)^{(q+1) / q}$. Then $E$ satisfies the differential inequality

$$
K_{1} \rho^{\delta \theta(q+1) / q} \frac{d E}{d \rho} \geq E(\rho)^{1+(1-\theta) / q-\tau(1-\theta)(q+1) / q(\sigma+1)}
$$

Integrating (2.25) yields (if $\tau(q+1)-\sigma-1>0$ )

$$
\begin{aligned}
& \frac{K_{1} q(\sigma+1)}{(1-\theta)(\tau(q+1)-\sigma-1)} \\
& \quad \times\left\{E\left(\rho_{0}\right)^{(1-\theta)(\tau(q+1)-\sigma-1) / q(\sigma+1)}-E\left(\rho_{1}\right)^{(1-\theta)(\tau(q+1)-\sigma-1) / q(\sigma+1)}\right\} \\
& \geq \frac{q}{q-\delta \theta(q+1)}\left(\rho_{0}^{1-\delta \theta(1+1 / q)}-\rho_{1}^{1-\delta \theta(1+1 / q)}\right) .
\end{aligned}
$$

Hence if

(2.27)

$$
\begin{aligned}
\rho_{1}^{1-\delta \theta(1+1 / q)}= & \rho_{0}^{1-\delta \theta(1+1 / q)} \\
& -\frac{K_{1}(\sigma+1)(q-\delta \theta(q+1))}{(1-\theta) /(\tau(q+1)-\sigma-1)} E\left(\rho_{0}\right)^{(1-\theta)(\tau(q+1)-\sigma-1) /(q(\sigma+1))},
\end{aligned}
$$

then $E\left(\rho_{1}\right)=0$ and $E(\rho)=0$ for $\rho \leq \rho_{1}$ so $(2.21)$ implies $b(\rho)=0$ which means $u(x)=0$ a.e. in $B_{\rho}\left(x_{0}\right)$ for $\rho \leq \rho_{1}$. If we compute the exponents we have

$$
1-\delta \theta\left(1+\frac{1}{q}\right)=\frac{\kappa}{q(\sigma+1)}, \quad \frac{(1-\theta)(\tau(q+1)-\sigma-1)}{q(\sigma+1)}=\frac{\tau(q+1)-\sigma-1}{\kappa},
$$

which implies (2.3) with (2.5) and (2.6).

REMARK 2.2. We can relax the hypotheses on $\alpha$ and $\beta$ in assuming that $u \in$ $L_{\text {loc }}^{\infty}(\Omega)$, which is the case if

$$
|C(x, r)| \leq C_{6}|r|^{q}+D
$$

$C_{6}$ and $D$ being some constants (see [26]). From (2.17) it is easy to see that we just have to suppose $\alpha \geq 0,0 \leq \beta \leq q+1$ and $C_{3}$ small enough in order to get (2.3).

As an application of Theorem 2.1 we have the following global result which contains Theorem I.

Corollary 2.2. Assume $\Omega=\mathbf{R}^{N}, C_{2}>0, C_{4}>0,0 \leq \sigma<q$, and (2.2) if $0<\beta<q+1$ or $C_{3}<C_{4}$ (resp. $C_{3}<C_{2}$ ) if $\beta=0$ (resp. $\beta=q+1$ ) and suppose $u \in W^{1, q+1}\left(\mathbf{R}^{N}\right) \cap L^{\sigma+1}\left(\mathbf{R}^{N}\right)$ is any weak solution of

$$
-\operatorname{div} A(x, u, D u)+B(x, u, D u)+C(x, u)=f(x)
$$


in $\mathbf{R}^{N}$, where $f \in L^{(\sigma+1) / \sigma}\left(\mathbf{R}^{N}\right)$. If $f$ has its support in $B_{\rho_{0}}(0)$, then there exists $\rho_{1}>\rho_{0}$ depending on $\|f\|_{L^{(\sigma+1) / \sigma}\left(\mathbf{R}^{N}\right)}$ and the structural constants $C_{1}, C_{2}, C_{3}, C_{4}$, $N, q, \sigma, \beta$ such that $\operatorname{supp}(u) \subset B_{\rho_{1}}(0)$. If we suppose moreover that $q+1<N$ or $C(x, r)$ satisfies $(2.28)$ the result remains true if we just suppose $u \in L^{\sigma+1}\left(\mathbf{R}^{N}\right)$, $D u \in L^{q+1}\left(\mathbf{R}^{N}\right)$ and $f \in L^{(q+1) / q}\left(\mathbf{R}^{N}\right)$.

PROOF. As $u$ is a weak solution of (2.29), we have

$\int_{\mathbf{R}^{N}} A(x, u, D u) \cdot D \varphi d x+\int_{\mathbf{R}^{N}} B(x, u, D u) \varphi d x+\int_{\mathbf{R}^{N}} C(x, u) \varphi d x=\int_{\mathbf{R}^{N}} f(x) \varphi d x$ for any $\varphi \in C_{0}^{\infty}\left(\mathbf{R}^{N}\right)$. Using the same truncation method as in Lemma 2.1 we have for any $\varsigma \in C_{0}^{\infty}\left(\mathbf{R}^{N}\right), \varsigma \geq 0$,

$$
\begin{aligned}
\int_{\mathbf{R}^{N}}\{\zeta A(x, u, D u) \cdot D u+u & \left.A(x, u, D u) \cdot D_{\zeta}+B(x, u, D u) u \zeta+C(x, u) u \zeta\right\} d x \\
= & \int_{\mathbf{R}^{N}} f u \zeta d x
\end{aligned}
$$

If we take $\zeta=\zeta_{n}$ such that $0 \leq \varsigma_{n} \leq 1, \zeta_{n}(x)=1$ if $|x| \leq n, \zeta_{n}(x)=0$ if $|x| \geq n+1$ and $\left\|D_{\zeta_{n}}\right\|_{L^{\infty}} \leq 2$, then

$$
\begin{aligned}
& \left|\int_{\mathbf{R}^{N}} u A(x, u, D u) \cdot D_{\zeta_{n}} d x\right| \\
& \quad \leq 2 C_{1}\left(\int_{n<|x|<n+1}|u|^{q+1} d x\right)^{1 /(q+1)}\left(\int_{n<|x|<n+1}|D u|^{q+1} d x\right)^{q /(q+1)} .
\end{aligned}
$$

If $n \rightarrow+\infty$ we deduce (as in the first step of the proof of Theorem 2.1)

$$
C_{5} \int_{\mathbf{R}^{N}}\left\{A(x, u, D u) \cdot D u+|u|^{\sigma+1}\right\} d x \leq \int_{\mathbf{R}^{N}} f u d x .
$$

From Young's inequality

$$
\int_{\mathbf{R}^{N}} f u d x \leq \varepsilon \int_{\mathbf{R}^{N}}|u|^{\sigma+1} d x+C_{\varepsilon} \int_{\mathbf{R}^{N}}|f|^{(\sigma+1) / \sigma} d x .
$$

If $\varepsilon<C_{5}$ we deduce that

$$
\int_{\mathbf{R}^{N}} A(x, u, D u) \cdot D u d x+\int_{\mathbf{R}^{N}}|u|^{\sigma+1} d x \leq k \int_{\mathbf{R}^{N}}|f|^{(\sigma+1) / \sigma} d x
$$

for some structural constant $k$. Hence $E(\infty)$ and $b(\infty)$ remain bounded independently of $u$ and, for any $r>1$ and $x_{0} \in \mathbf{R}^{N}$,

$$
C \min _{1 /(q+1)<\tau \leq 1}\left\{\frac{E^{\gamma}(r)}{\tau(q+1)-1} \max \left(1, r^{\nu-1}\right) \max \left(b^{\mu}(r), b^{\eta}(r)\right)\right\} \leq K r^{\nu-1}
$$

where $C$ depends on the structural constants and $\int_{\mathbf{R}^{N}}|f|^{(\sigma+1) / \sigma} d x$. If we apply Theorem 2.1 in $B_{r}\left(x_{0}\right)$, where $\left|x_{0}\right|=\rho_{0}+r$, we deduce that supp $u \subset B_{\rho_{1}}(0)$ with $\rho_{1}=\rho_{0}+\max (1, K)$.

If we suppose $q+1<N$, then $D u \in L^{q+1}\left(\mathbf{R}^{N}\right)$ implies $u \in L^{(q+1) *}\left(\mathbf{R}^{N}\right)$ with

$$
\frac{1}{(q+1)^{*}}=\frac{1}{q+1}-\frac{1}{N}
$$


Hence $u \in L^{\sigma+1}\left(\mathbf{R}^{N}\right) \cap L^{q+1}\left(\mathbf{R}^{N}\right)$, so $u \in W^{1, q+1}\left(\mathbf{R}^{N}\right)$ and we can go to the limit in (2.32) and get (2.34). If $C(x, r)$ satisfies $(2.28)$, then $u \in L^{\infty}\left(\mathbf{R}^{N}-B_{2 \rho_{0}}(0)\right)$ (see [26]) and $u \in L^{q+1}\left(\mathbf{R}^{N}\right)$ from interpolation results.

REMARK 2.3. The result of Corollary 2.2 is already known when (2.29) has the particular form

$$
-\operatorname{div}\left(|D u|^{q-1} D u\right)+|u|^{\sigma-1} u=f
$$

(see $[\mathbf{8}, \mathbf{1 6}, 34])$; it fails if $q \leq \sigma$. Other vanishing properties for first order quasilinear equations of the form

$$
-\sum_{i} \frac{\partial}{\partial x_{i}}\left(\Phi_{i}(u)\right)+\beta(u)=f
$$

can be found in $[\mathbf{1 7}]$.

REMARK 2.4. In Theorem 2.1 we can relax the hypothesis of continuity on $R \mapsto C(x, r)$ in order to treat some variational inequalities $(\sigma=0)$. We can also deal with unilateral constraints on $u$ such as the weak variational inequality

$$
\left\{\begin{array}{l}
u \geq 0, \\
-\operatorname{div} A(x, u, D u)+B(x, u, D u) \geq f(x),
\end{array}\right.
$$

in the sense that $u \in L^{1}(\Omega), D u \in L^{q+1}(\Omega), B(\cdot, u, D u) \in L^{1}(\Omega)$ and

$$
\int_{\Omega}\{A(x, u, D u) \cdot D \varphi+B(x, u, D u) \varphi\} d x \geq \int_{\Omega} f(x) \varphi d x
$$

for any $\varphi \in C_{0}^{\infty}\left(\mathbf{R}^{N}\right), \varphi \geq 0$. If for some $\varepsilon>0$ we have $f(x) \leq-\varepsilon$ a.e. in $\Omega$ we can apply Theorem 2.1 provided $C_{3}$ is small enough (see [11] for a basic result).

3. The parabolic equation. In this section $\Omega$ is an open subset of $\mathbf{R}^{N}, N \geq 1$, $A, B$ and $C$ are Carathéodory vector valued (for $A$ ) or real valued (for $B$ and $C$ ) functions defined in $\mathbf{R}^{+} \times \Omega \times \mathbf{R} \times \mathbf{R}^{N}$ (for $A$ and $B$ ) or in $\mathbf{R}^{+} \times \Omega \times \mathbf{R}$ (for $C$ ) satisfying (P1)-(P4) and $\psi$ satisfies (P5).

DEFINITION 3.1. A measurable function $u$ defined in $\mathbf{R}^{+} \times \Omega$ is a weak solution of (PE) with initial data $u_{0}$ if for any $T>0$ and any open subset $G$ relatively compact in $\Omega$ we have

(i) $D u \in L^{q+1}((0, T) \times G)$,

(ii) $B(\cdot, \cdot, u, D u) \in L^{1}((0, T) \times G)$,

(iii) $C(\cdot, \cdot, u) \in L^{1}((0, T) \times G)$,

(iv) $j(u(t, \cdot)) \in L^{\infty}\left(0, T ; L^{1}(G)\right)$,

(v) $\lim \operatorname{ess}_{t \downarrow 0} j(u(t, \cdot))=j\left(u_{0}(\cdot)\right)$ in $L^{1}(G)$,

where $j(r)=\int_{0}^{r} \psi(s) d s$ satisfies the $\Delta_{2}$ condition and for any $\varsigma \in C_{0}^{\infty}\left(\mathbf{R}^{+} \times \mathbf{R}^{N}\right)$ we have

$$
\begin{aligned}
\int_{0}^{+\infty} & \int_{\Omega}\{A(s, x, u, D u) \cdot D \zeta+B(s, x, u, D u) \zeta+C(s, x, u) \zeta\} d x d s \\
= & \int_{0}^{+\infty} \int_{\Omega} \psi(u(s, x)) \frac{\partial \zeta}{\partial t} d x d s+\int_{\Omega} \psi\left(u_{0}(x)\right) \zeta(0, x) d x
\end{aligned}
$$

Our first result is a finite speed of propagation property of the support of the weak solutions of (PE). 
THEOREM 3.1. Suppose $M_{2}>0, M_{3} \geq 0, M_{4}=0, m q>1, c>0,0 \leq \beta \leq$ $q+1, \alpha=(q+1-\beta(m+1)) / m(q+1)$ and let $T^{*}$ be $c / M_{3}\left(\right.$ resp. $+\infty$ and $\left.M_{3}<M_{2}\right)$ if $\beta=0$ (resp. $\beta=q+1)$ or

$$
T^{*}=\frac{c(q+1)}{q+1-\beta} M_{3}^{-(q+1) /(q+1-\beta)}\left(M_{2} \frac{q+1}{\beta}\right)^{\beta /(q+1-\beta)}
$$

if $0<\beta<q+1$. If $u$ is a weak solution of $(\mathrm{PE})$ in $\mathbf{R}^{+} \times \Omega$ with an initial data $u_{0}$ vanishing in $B_{\rho_{0}}\left(x_{0}\right), x_{0} \in \Omega, 0<\rho_{0}<\operatorname{dist}\left(x_{0}, \partial \Omega\right)$, then $u(t, \cdot)=0$ a.e. in $B_{\rho_{1}(t)}\left(x_{0}\right)$ for $0 \leq t \leq T$, where $T<T^{*}$ is arbitrary,

$$
\begin{array}{r}
\rho_{1}^{\nu}(t)=\rho_{0}^{\nu}-C t^{\lambda} \min _{(m+1) /(m(q+1))<\tau \leq 1}\left\{\frac{E^{\gamma}\left(t, \rho_{0}\right)}{m \tau(q+1)-m-1} \max \left(1, \rho_{0}^{\nu-1}\right)\right. \\
\left.\times \max \left(b^{\mu}\left(t, \rho_{0}\right), b^{\eta}\left(t, \rho_{0}\right)\right)\right\}
\end{array}
$$

where $C=C\left(M_{1}, M_{2}, M_{3}, N, q, m, \beta, T, c\right)$ and

$$
\begin{gathered}
E(t, \rho)=\int_{0}^{t} \int_{B_{\rho}\left(x_{0}\right)} A(\tau, x, u, D u) \cdot D u d x d \tau, \\
b(t, \rho)=\sup _{0 \leq \tau \leq t} \int_{B_{\rho}\left(x_{0}\right)}|u(\tau, x)|^{(m+1) / m} d x \\
\kappa=N(m q-1)+(q+1)(m+1), \\
\gamma=\frac{m \tau(q+1)-m-1}{\kappa}, \quad \mu=\frac{m(1-\tau)(q+1)}{\kappa}, \\
\nu=\frac{\kappa}{q(m+1)}, \quad \lambda=\frac{m+1}{\kappa}, \quad \eta=\frac{m q-1}{q(m+1)}-\frac{m+1-m \tau(q+1)}{\kappa} .
\end{gathered}
$$

REMARK 3.1. As in Theorem 2.1, $B_{\rho_{1}(t)}\left(x_{0}\right)=\varnothing$ if $\rho_{1}(t) \leq 0$, but for $t$ small enough $\rho_{1}(t)$ is positive, which means that the speed of propagation of the support of $u$ is finite. Moreover if the function $t \mapsto j(u(t, \cdot))$ is essentially continuous from $\mathbf{R}^{+}$into $L_{\text {loc }}^{1}(\Omega)$ we can iterate Theorem 3.1 on $(T, 2 T),(2 T, 3 T)$, etc., for a.e. $T$, as long as $\rho_{1}$ remains $>0$ : we just have to replace $t$ by $t-T$ (on $(T, 2 T))$. An exact formula for the speed of the interface is difficult to obtain as $\lim _{t \rightarrow 0} b\left(t, \rho_{0}\right)=\lim _{t \rightarrow 0} E\left(t, \rho_{0}\right)=0$ (see Knerr [25] for computations in the porous medium equation case).

LEMMA 3.1. Under the hypotheses of Theorem 3.1, $A(\cdot, \cdot, u, D u) \cdot D u$, $B(\cdot, \cdot, u, D u) u, C(\cdot, \cdot, u) u$ and $u|A(\cdot, \cdot, u, D u)|$ belong to $L^{1}\left((0, T) \times B_{\rho_{0}}\left(x_{0}\right)\right)$ and for almost all $\rho \in\left(0, \rho_{0}\right)$ and $t \in\left(0, T^{*}\right)$ the following inequality holds:

$$
\begin{aligned}
\int_{0}^{t} \int_{B_{\rho}\left(x_{0}\right)}\{A(\tau, x, u, D u) \cdot D u+B(\tau, x, u, D u) u+C(\tau, x, u) u\} d x d \tau \\
\quad+\int_{B_{\rho}\left(x_{0}\right)} f(u(t, x)) d x \\
\quad \leq \int_{B_{\rho}\left(x_{0}\right)} f\left(u_{0}(x)\right) d x+\int_{0}^{t} \int_{S_{\rho}\left(x_{0}\right)} A(\tau, x, u, D u) \cdot \vec{\nu} u d s d \tau .
\end{aligned}
$$

Proof. From hypotheses it is clear that $A(\cdot, \cdot, u, D u) \cdot D u, B(\cdot, \cdot, u, D u) u$ and $u|A(\cdot, \cdot, u, D u)|$ are locally integrable in $\mathbf{R}^{+} \times \Omega$. We now define for $l \in \mathbf{N}, T_{l}(u)=$ 
$\operatorname{sign}(u) \min (l,|u|)$. For $n \in \mathbf{N}$ and $\rho \in\left(0, \rho_{0}\right)$ we consider $\psi_{n}$ defined on $\left[0, \rho_{0}\right]$ by

$$
\psi_{n}(r)= \begin{cases}1 & \text { if } r \in[0, \rho-1 / n], \\ 0 & \text { if } r \in\left[\rho, \rho_{0}\right], \\ -n(\rho-r) & \text { if } r \in[\rho-1 / n, \rho] .\end{cases}
$$

For $t \in\left(0, T^{*}\right)$ and $k \in \mathbf{N}$ set $\zeta_{k}$ defined on $\left[0, T^{*}\right]$ by

$$
\zeta_{k}(r)= \begin{cases}1 & \text { if } r \in[0, t-1 / k], \\ 0 & \text { if } r \in\left[t, T^{*}\right], \\ -k(t-r) & \text { if } r \in[t-1 / k, t] .\end{cases}
$$

For $h \in\left(0, T^{*}-t\right)$ we set

$$
\varphi(\tau, x)=\varphi_{n, l, k, h}(\tau, x)=\zeta_{k}(\tau) \psi_{n}\left(\left|x-x_{0}\right|\right) \int_{\tau}^{\tau+h} h^{-1} T_{l}(u(\varepsilon, x)) d \varepsilon .
$$

It is clear that $\varphi$ is an admissible test function so we have

$$
\begin{aligned}
\int_{0}^{+\infty} & \int_{B_{\rho}\left(x_{0}\right)}\{A(\tau, x, u, D u) \cdot D \varphi+B(\tau, x, u, D u) u+C(\tau, x, u) u\} d x d \tau \\
= & \int_{0}^{\infty} \int_{B_{\rho_{0}}\left(x_{0}\right)} \psi(u(\tau, x)) \frac{\partial \varphi}{\partial \tau} d x d \tau+\int_{B_{\rho_{0}}\left(x_{0}\right)} \psi\left(u_{0}\right) \varphi(0, x) d x
\end{aligned}
$$

From (iv) it is clear that both $\psi(u)$ and $u \psi(u)$ belong to $L^{\infty}\left(0, T^{*} ; L^{1}\left(B_{\rho_{0}}\left(x_{0}\right)\right)\right.$ and it is the same with the function $\tau \mapsto \psi(u(\tau, \cdot)) \int_{\tau}^{\tau+h} h^{-1} T_{l}(u(\varepsilon, \cdot)) d \varepsilon$ so we can suppose that $t$ is one of its Lebesgue points (independently of $l$ and $h$ ). Hence

$$
\begin{aligned}
\lim _{k \rightarrow+\infty} & \int_{0}^{+\infty} \int_{B_{\rho_{0}}\left(x_{0}\right)} \psi(u(\tau, x)) \frac{\partial \varphi}{\partial \tau} d x d \tau \\
\quad= & \int_{0}^{t} \int_{B_{\rho_{0}}\left(x_{0}\right)} \psi(u(\tau, x)) \frac{\partial \tilde{\varphi}}{\partial \tau} d x d \tau-\int_{B_{\rho_{0}}\left(x_{0}\right)} \psi(u(t, x)) \tilde{\varphi}(t, x) d x
\end{aligned}
$$

where $\tilde{\varphi}(\tau, x)=\tilde{\varphi}_{n, l, h}(\tau, x)=\psi_{n}\left(\left|x-x_{0}\right|\right) \int_{\tau}^{\tau+h} h^{-1} T_{l}(u(\varepsilon, x)) d \varepsilon$. So (3.13) becomes

$$
\begin{aligned}
& \int_{0}^{t} \int_{B_{\rho_{0}}\left(x_{0}\right)}\{A(\tau, x, u, D u) \cdot D \tilde{\varphi}+B(\tau, x, u, D u) \tilde{\varphi}+C(\tau, x, u) \tilde{\varphi}\} d x d \tau \\
& \quad=\int_{0}^{t} \int_{B_{\rho_{0}}\left(x_{0}\right)} \psi(u(\tau, x)) \frac{\partial \tilde{\varphi}}{\partial \tau} d x d \tau-\left[\int_{B_{\rho_{0}}\left(x_{0}\right)} \psi(u(\tau, x)) \tilde{\varphi}(\tau, x) d x\right]_{\tau=0}^{\tau=t} .
\end{aligned}
$$

As $j$ is convex and increasing on $\mathbf{R}^{+}$(resp. decreasing on $\mathbf{R}^{-}$) we have (3.15) $j\left(T_{l}(u(\tau+h, x))\right)-j\left(T_{l}(u(\tau, x))\right) \geq \psi(u(\tau, x))\left(T_{l}(u(\tau+h, x))-T_{l}(u(\tau, x))\right)$.

So computing $\partial \tilde{\varphi} / \partial \tau$ and using (3.15) yields

$$
\begin{aligned}
& \int_{0}^{t} \int_{B \rho_{0}\left(x_{0}\right)} \psi(u(\tau, x)) \frac{\partial \tilde{\varphi}}{\partial \tau} d x d \tau \\
& \quad \leq h^{-1} \int_{t}^{t+h} \int_{B_{\rho_{0}}\left(x_{0}\right)} j\left(T_{l} u\right) \psi_{n} d x d \tau-h^{-1} \int_{0}^{h} \int_{B_{\rho_{0}}\left(x_{0}\right)} j\left(T_{l} u\right) \psi_{n} d x d \tau
\end{aligned}
$$


where $\psi_{n}(\cdot)=\psi_{n}\left(\left|\cdot-x_{0}\right|\right)$. Using again the properties of $j$ and (v) implies that the right-hand side on (3.16) converges to

$$
\int_{B_{\rho_{0}}\left(x_{0}\right)} j\left(T_{l} u(t, x)\right) \psi_{n}(x) d x-\int_{B_{\rho_{0}}\left(x_{0}\right)} j\left(T_{l} u_{0}(x)\right) \psi_{n}(x) d x
$$

as $h$ goes to 0 , for almost all $t$. If we set

$$
\varphi_{n, l}(\tau, x)=\psi_{n}\left(\left|x-x_{0}\right|\right) T_{l}(u(\tau, x))
$$

we deduce with Lebesgue and Fatou's theorems (as $h \rightarrow 0$ )

$$
\begin{gathered}
\int_{0}^{t} \int_{B_{\rho_{0}}\left(x_{0}\right)}\left\{A(\tau, x, u, D u) \cdot D \varphi_{n, l}+B(\tau, x, u, D u) \varphi_{n, l}+C(\tau, x, u) \varphi_{n, l}\right\} d x d \tau \\
\leq\left[\int_{B_{\rho_{0}}\left(x_{0}\right)}\left\{j\left(T_{l}(u(\tau, x))\right)-\psi(u(\tau, x)) T_{l}(u(\tau, x))\right\} \psi_{n}\left(\left|x-x_{0}\right|\right) d x\right]_{\tau=0}^{\tau=t} .
\end{gathered}
$$

From hypotheses the right-hand side of $(3.18)$ converges to

$$
\left[\int_{B_{\rho_{0}}\left(x_{0}\right)} f(u(\tau, x)) \psi_{n}\left(\left|x-x_{0}\right|\right) d x\right]_{\tau=t}^{\tau=0}
$$

as $l$ goes to $+\infty$. Moreover, computing $D \psi_{n}$ and using Lebesgue's differentiation theorem as in Lemma 2.1 yield for a.e. $\rho \in\left(0, \rho_{0}\right)$

$$
\begin{aligned}
\lim _{n \rightarrow \infty} & -\int_{0}^{t} \int_{B_{\rho}\left(x_{0}\right)} u \mathcal{A}(\tau, x, u, D u) \cdot D \psi_{n} d x d \tau \\
& =\int_{0}^{t} \int_{S_{\rho}\left(x_{0}\right)} u \mathcal{A}(\tau, x, u, D u) \cdot \vec{\nu} d s d \tau
\end{aligned}
$$

Moreover $\int_{0}^{t} \int_{B_{\rho_{0}}\left(x_{0}\right)} C(\tau, x, u) \cdot u d x d \tau$ exists and we have

$$
\begin{aligned}
& \int_{0}^{t} \int_{B_{\rho}\left(x_{0}\right)}\{A(\tau, x, u, D u) \cdot D u+B(\tau, x, u, D u) u+C(\tau, x, u) u\} d x d \tau \\
& \leq \int_{0}^{t} \int_{S_{\rho}\left(x_{0}\right)} u A(\tau, x, u, D u) \cdot \vec{\nu} d s d \tau+\left[\int_{B_{\rho}\left(x_{0}\right)} f(u(\tau, x)) d x\right]_{\tau=t}^{\tau=0},
\end{aligned}
$$

which is (3.9).

Proof of ThEOREM 3.1. First step. We fix $T<T^{*}$ and we claim that there exists a constant $M_{5}=M_{5}\left(T, M_{2}, M_{4}, m, c, q, \beta\right), M_{5}>0$, such that for any $(t, \rho) \in[0, T] \times\left[0, \rho_{0}\right]$ the following holds:

$$
\begin{aligned}
& E(t, \rho)+c b(t, \rho)-\left|\int_{0}^{t} \int_{B_{\rho}\left(x_{0}\right)} u B(\tau, x, u, D u) d x d \tau\right| \\
& \leq M_{5}(E(t, \rho)+b(t, \rho)) .
\end{aligned}
$$

This is clear when $\beta=0$ or $\beta=q+1$. When $0<\beta<q+1$ we have

$$
\left|\int_{0}^{t} \int_{B_{\rho}\left(x_{0}\right)} u B(\tau, x, u, D u) d x d \tau\right| \leq M_{3} \int_{0}^{t} \int_{B_{\rho}\left(x_{0}\right)}|u|^{\alpha+1}|D u|^{\beta} d x d \tau
$$


and with Young's inequality we get for any $\varepsilon>0$ and $\tau \in(1,+\infty)$

$$
|u|^{\alpha+1}|D u|^{\beta} \leq \frac{\varepsilon}{\tau}|u|^{\tau(\alpha+1)}+\frac{(\tau-1)}{\tau} \varepsilon^{-1 /(\tau-1)}|D u|^{\beta \tau /(\tau-1)} .
$$

If we choose $\tau=(m+1) / m(\alpha+1)$, then $\beta \tau /(\tau-1)=q+1$ and

$$
\begin{gathered}
\left|\int_{0}^{t} \int_{B_{\rho}\left(x_{0}\right)} B(\tau, x, u, D u) u d x d \tau\right| \\
\leq \varepsilon M_{3} \frac{q+1-\beta}{q+1} \int_{0}^{t} \int_{B_{\rho}\left(x_{0}\right)}|u|^{(m+1) / m} d x d t \\
\quad+\frac{\beta M_{3}}{M_{2}(q+1)} \varepsilon^{-(q+1-\beta) / \beta} E(t, \rho) .
\end{gathered}
$$

But

$$
\int_{0}^{t} \int_{B_{\rho}\left(x_{0}\right)}|u|^{(m+1) / m} d x d \tau \leq T b(t, \rho) .
$$

As $T<T^{*}$ it is possible to find $\varepsilon$ depending on $T, M_{2}, M_{4}, m, q, c$ and $\beta$ such that

$$
\varepsilon M_{3} T \frac{q+1-\beta}{q+1}<c \text { and } \frac{\beta M_{3}}{M_{2}(q+1)} \varepsilon^{-(q+1-\beta) / \beta}<1 .
$$

If we set

$$
M_{5}=\min \left\{c-\varepsilon M_{3} T \frac{q+1-\beta}{q+1}, 1-\frac{\beta M_{3}}{M_{2}(q+1)} \varepsilon^{-(q+1-\beta) / \beta}\right\}
$$

we get (3.22).

End of the proof. From (3.9) we have for $(t, \rho) \in[0, T] \times\left[0, \rho_{0}\right]$,

$$
\begin{gathered}
E(t, \rho)+c \int|u(t, x)|^{(m+1) / m} d x-\left|\int_{0}^{t} \int_{B_{\rho}\left(x_{0}\right)} B(\tau, x, u, D u) u d x d \tau\right| \\
\leq \int_{B_{\rho}\left(x_{0}\right)} f\left(u_{0}(x)\right) d x+\int_{0}^{t} \int_{S_{\rho}\left(x_{0}\right)} A(\tau, x, u, D u) \cdot \vec{\nu} u d s d \tau
\end{gathered}
$$

But $u_{0}$ vanishes in $B_{\rho}\left(x_{0}\right)$, and from Hölder's inequality and (P1)

$$
\begin{aligned}
& \int_{0}^{t} \int_{S_{\rho}\left(x_{0}\right)} A(\tau, x, u, D u) \cdot \vec{\nu} u d s d \tau \\
& \quad \leq M_{1}\left(\int_{0}^{t} \int_{S_{\rho}\left(x_{0}\right)}|D u|^{q+1} d s d \tau\right)^{q /(q+1)}\left(\int_{0}^{t} \int_{S_{\rho}\left(x_{0}\right)}|u|^{q+1} d s d \tau\right)^{1 /(q+1)}
\end{aligned}
$$

As in the proof of Theorem 2.1 we have for almost all $\rho \in\left[0, \rho_{0}\right]$,

$$
\frac{\partial E}{\partial \rho}(t, \rho) \geq M_{2} \int_{0}^{t} \int_{S_{\rho}\left(x_{0}\right)}|D u|^{q+1} d s d \tau .
$$


Using (3.22), (3.26), (3.27) and (3.28) we get

(3.29)

$$
\begin{aligned}
& M_{5}(E(t, \rho)+b(t, \rho)) \\
& \quad \leq 2 M_{1} M_{2}^{-q /(q+1)}\left(\frac{\partial E}{\partial \rho}(t, \rho)\right)^{q /(q+1)}\left(\int_{0}^{t} \int_{S_{\rho}\left(x_{0}\right)}|u|^{q+1} d s d \tau\right)^{1 /(q+1)} .
\end{aligned}
$$

Using (2.13) with $\sigma$ replaced by $1 / m$ (which is possible as $q m>1$ ) we deduce

$$
\begin{aligned}
\int_{0}^{t} & \int_{S_{\rho}\left(x_{0}\right)}|u|^{q+1} d s d \tau \\
& \leq C^{q+1} \int_{0}^{t}\left(\|D u\|_{L^{q+1}\left(B_{\rho}\left(x_{0}\right)\right)}+\rho^{\delta}\|u\|_{L^{(m+1) / m}\left(B_{\rho}\left(x_{0}\right)\right)}\right)^{\theta(q+1)} \\
& \times\|u\|_{L^{(m+1) / m}\left(B_{\rho}\left(x_{0}\right)\right)}^{(1-\theta)(q+1)} d \tau
\end{aligned}
$$

with

$$
\theta=\frac{N(m q-1)+m+1}{N(m q-1)+(m+1)(q+1)} \quad \text { and } \quad \delta=-\frac{N(m q-1)+(m+1)(q+1)}{(m+1)(q+1)} .
$$

Using Hölder's inequality and $(a+b)^{q+1} \leq 2^{q+1}\left(a^{q+1}+b^{q+1}\right)$, we get

$$
\begin{aligned}
& \int_{0}^{t} \int_{S_{\rho}\left(x_{0}\right)}|u|^{q+1} d s d \tau \\
& \leq(2 C)^{q+1}\left(\int_{0}^{t} \int_{B_{\rho}\left(x_{0}\right)}|D u|^{q+1} d x d \tau\right. \\
& \left.+\cdots+\rho^{\delta(q+1)} \int_{0}^{t}\|u\|_{L^{(m+1) / m}\left(B_{\rho}\left(x_{0}\right)\right)}^{q+1} d \tau\right)^{\theta} \\
& \quad \times\left(\int_{0}^{t}\|u\|_{L^{(m+1) / m}\left(B_{\rho}\left(x_{0}\right)\right)}^{q+1} d \tau\right)^{1-\theta}
\end{aligned}
$$

which implies

$$
\begin{aligned}
& \left(\int_{0}^{t} \int_{S_{\rho}\left(x_{0}\right)}|u|^{q+1} d s d \tau\right)^{1 /(q+1)} \\
& \leq 2 C t^{(1-\theta) /(q+1)}\left(\|D u\|_{L^{q+1}\left((0, t) \times B_{\rho}\left(x_{0}\right)\right)}\right. \\
& \left.\quad+\rho^{\delta} t^{1 /(q+1)} b^{m /(m+1)}(t, \rho)\right)^{\theta} b^{m(1-\theta) /(m+1)}(t, \rho) .
\end{aligned}
$$

Then there exists a constant $K=K\left(T, M_{2}, M_{4}, m, q, \beta\right)$ such that

$$
\begin{aligned}
E(t, \rho)+b(t, \rho) \leq & K t^{(1-\theta) /(q+1)}\left(\frac{\partial E}{\partial \rho}(t, \rho)\right)^{q /(q+1)} \\
& \times\left(E^{1 /(q+1)}(t, \rho)+\rho^{\delta} t^{1 /(q+1)} b^{m /(m+1)}(t, \rho)\right)^{\theta} b^{m(1-\theta) /(m+1)}(t, \rho) .
\end{aligned}
$$


For any $0 \leq \tau \leq 1$ we have

$$
\begin{aligned}
& E^{1 /(q+1)}(t, \rho) b^{m(1-\theta) / \theta(m+1)}(t, \rho)+\rho^{\delta} t^{1 /(q+1)} b^{m / \theta(m+1)}(t, \rho) \\
&= E^{1 /(q+1)}(t, \rho) b^{\tau m(1-\theta) / \theta(m+1)}(t, \rho) b^{(1-\tau)(1-\theta) m / \theta(m+1)}(t, \rho) \\
&+\rho^{\delta} t^{1 /(q+1)} b^{1 /(q+1)+\tau m(1-\theta) / \theta(m+1)}(t, \rho) \\
& \times(t, \rho)^{m / \theta(m+1)-1 /(q+1)-\tau m(1-\theta) / \theta(m+1)}(t, \rho) .
\end{aligned}
$$

We set

$$
\begin{aligned}
K_{0}=\max \left(1, T^{\theta /(q+1)}\right) \max \left(b^{m(1-\tau)(1-\theta) /(m+1)}\left(t, \rho_{0}\right),\right. & \\
& \left.b^{(m-m \tau(1-\theta)) /(m+1)-\theta /(q+1)}\left(t, \rho_{0}\right)\right)
\end{aligned}
$$

and get

$$
\begin{aligned}
& E^{1 /(q+1)}(t, \rho) b^{m(1-\theta) / \theta(m+1)}(t, \rho)+\rho^{\delta} t^{1 /(q+1)} b^{m / \theta(m+1)}(t, \rho) \\
& \quad \leq 2 \rho^{\delta} K_{0}^{1 / \theta} \max \left(1, \rho_{0}^{-\delta}\right)(E(t, \rho)+b(t, \rho))^{1 /(q+1)+m \tau(1-\theta) /(m+1)} .
\end{aligned}
$$

This implies the inequality

$$
\begin{aligned}
& (E(t, \dot{\rho})+b(t, \rho))^{1-\theta /(q+1)-m \tau(1-\theta) /(m+1)} \\
& \quad \leq 2 K t^{(1-\theta) /(q+1)} \rho^{\delta \theta} K_{0} \max \left(1, \rho_{0}^{-\delta \theta}\right)\left(\frac{\partial E}{\partial \rho}(t, \rho)\right)^{q /(q+1)},
\end{aligned}
$$

and finally $E$ satisfies the differential inequality

$$
K_{1} t^{(1-\theta) / q} \rho^{\delta \theta(1+1) / q} \frac{\partial E}{\partial \rho}(t, \rho) \geq E^{1+(1-\theta) / q-\tau m(1-\theta)(q+1) / q(m+1)}(t, \rho),
$$

with $K_{1}=\left(2 K K_{0} \max \left(1, \rho_{0}^{-\delta \theta}\right)\right)^{(q+1) / q}$. Integrating (3.34) yields

$$
\begin{aligned}
& \frac{(m+1) q K_{1} t^{(1-\theta) / q}}{(1-\theta)(m \tau(q+1)-m-1)} \\
& \quad \cdots\left\{E^{(1-\theta)(m \tau(q+1)-m-1) / q(m+1)}\left(t, \rho_{0}\right)\right. \\
& \left.\quad-E^{(1-\theta)(m \tau(q+1)-m-1) / q(m+1)}\left(t, \rho_{1}\right)\right\} \\
& \geq \frac{q}{q-\delta \theta(q+1)}\left(\rho_{0}^{1-\delta \theta(1+1 / q)}-\rho_{1}^{1-\delta \theta(1+1 / q)}\right),
\end{aligned}
$$

and the end of the proof is as in Theorem 2.1.

REMARK 3.2. As in Theorem 2.1 it is possible to relax the hypotheses on $\alpha$ and $\beta$ if we know a priori that $u \in L^{\infty}\left((0, T) \times B_{\rho_{0}}\left(x_{0}\right)\right)$. We just have to assume $\alpha \geq 0$, $0 \leq \beta \leq q+1$ and $M_{3}$ small enough (cf. [35] for some specific $L^{\infty}$ estimates).

COROLlary 3.1. Assume that $u$ is a weak solution of

$$
\frac{\partial}{\partial t} \psi(u)-\operatorname{div} A(t, x, u, D u)+B(t, x, u, D u)+C(t, x, u)=0
$$

in $\mathbf{R}^{+} \times \mathbf{R}^{N}$ such that $j(u) \in C^{0}\left(\mathbf{R}^{+} ; L_{\text {loc }}^{1}\left(\mathbf{R}^{N}\right)\right)$ and that for any $\rho>0, t>0$ there exists $K=K(t, \rho)$ such that for any $y \in \mathbf{R}^{N}$

$$
\underset{\tau \leq t}{\sup \operatorname{ess}} \int_{B_{\rho}(y)} f(u(\tau, x)) d x+\int_{0}^{t} \int_{B_{\rho}(y)} \mathcal{A}(\tau, x, u, D u) \cdot D u d x d \tau \leq K,
$$


and assume also that the structural hypotheses of Theorem 3.1 are satisfied (with $M_{3}<M_{2}$ if $\left.\beta=q+1\right)$. If the initial data $u_{0}$ of $u$ vanishes outside $B_{R}(0)$, then there exists a nondecreasing function $t \mapsto R(t)$ defined on $\mathbf{R}^{+}$such that $R(0)=R$ and $\operatorname{supp} u(t, \cdot) \subset B_{R(t)}(0)$ for any $t \geq 0$. If $B=0$ or $\beta=q+1$, then

$$
R(t)=R+C \max \left(t^{\lambda}, t^{1 / q}\right)
$$

with $\lambda$ given in equation (3.8) and $C$ depends on the structural constants, and $\left\|u_{0}\right\|_{L^{(m+1) / m}\left(\mathbf{R}^{N}\right)}$.

Proof. Step 1. We claim that there exists $T>0$ such that for any $t \in[0, T]$ the support of $u(t, \cdot)$ is compact.

In order to prove this result, we fix $T^{\prime}<T^{*}, \rho>0$ and $\left|x_{0}\right|>R+\rho$ and apply Theorem 3.1 in $B_{\rho}\left(x_{0}\right)$. From (3.37) there exists a constant $M$ depending on the structural constants, $T^{\prime}$ and $\rho$ but not on $x_{0}$ such that if $\rho^{\nu} \geq M t^{\lambda} \max \left(1, \rho^{\nu-1}\right)$ and $t<T^{\prime}$, then $u(t, x)=0$ a.e. in $B_{\rho_{1}(t)}\left(x_{0}\right)$, where $\rho_{1}^{\nu}(t)=\rho^{v}-M t^{\lambda} \max \left(1, \rho^{\nu-1}\right)$. If we set $T^{\lambda}=\min \left(T^{\prime \lambda}, \min \left(\rho^{\nu}, \rho\right) / M\right)$ and make $x_{0}$ run all over the complementary of $B_{R+\rho}(0)$ we deduce that for any $t \leq T, u(t, x)$ vanishes for almost all $|x|>$ $R+\rho-\rho_{1}(t)$.

Step 2. We claim that for any $t>0$ the support of $u(t, \cdot)$ is compact.

We proceed by contradiction in supposing that the subset of the $t$ 's of $\mathbf{R}^{+}$such that the support of $u(\tau, \cdot)$ is compact for $0 \leq \tau<t$ admits an upper bound $t^{*}<+\infty$. From (3.37) we have

$$
\sup _{\tau \leq 2 t^{*}} \int_{B_{\rho}(y)} f(u(\tau, x)) d x+\int_{0}^{2 t^{*}} \int_{B_{\rho}(y)} A(\tau, x, u, D u) \cdot D u d x d \tau \leq K\left(t^{*}, \rho\right)
$$

for any $y \in \mathbf{R}^{N}$. For any $t<t^{*}$ the support of $u(t, \cdot)$ is included in some ball $B_{R(t)}(0)$, so we can apply Theorem 3.1 on $[t,+\infty) \times \mathbf{R}^{N}$ (if we set $s=\tau-t$ and $v(s, x)=u(\tau, x)$ the function $v$ satisfies (PE) in $\mathbf{R}^{+} \times \mathbf{R}^{N}$ with $u(t, \cdot)$ as an initial data). Proceeding as in Step 1 we see that there exists $M>0$ such that, for any $|y|>R(t)+\rho$ and $(\tau-t)^{\lambda} \leq \min \left(t^{* \lambda}, \min \left(\rho^{\nu}, \rho\right) / M\right), u(\tau, \cdot)$ vanishes a.e. in $B_{\rho(\tau)}(y)$ where $\rho^{\nu}(\tau)=\rho^{\nu}-M(\tau-t) \max \left(1, \rho^{\nu-1}\right)$. Moreover from (3.39) and the definition of $v$ the constant $M$ does not depend on $t<t^{*}$ and on $y$ in $\mathbf{R}^{N}-B_{R(t)+\rho}(0)$, so $u(\tau, x)=0$ for almost all $|x|>R+\rho-\rho(\tau)$. In particular for

$$
\tau=\min \left(t^{*}+t, t+\left(\frac{1}{M} \min \left(\rho^{\nu}, \rho\right)\right)^{1 / \lambda}\right)
$$

$u(\tau, \cdot)$ vanishes a.e. in $\mathbf{R}^{N}-B_{R+\rho-\rho(\tau)}(0)$. If we take $t$ close enough to $t^{*}$ we have a contradiction, so $t^{*}=+\infty$. Moreover from the construction there exists a nondecreasing function $R$ defined on $\mathbf{R}^{+}$such that $R(0)=R$ and $\operatorname{supp} u(t, \cdot) \subset$ $B_{R(t)}(0)$. $t \geq 0$,

Step 3. End of the proof. If we apply Lemma 3.1 in $[0, t] \times B_{2 R(t)}(0)$, we get for

$$
\begin{gathered}
\int_{\mathbf{R}^{N}} f(u(t, x)) d x+\int_{0}^{t} \int_{\mathbf{R}^{N}}\{A(\tau, x, u, D u) \cdot D u+B(\tau, x, u, D u) u\} d x d \tau \\
\leq \int_{\mathbf{R}^{N}} f\left(u_{0}(x)\right) d x
\end{gathered}
$$


If $B=0$ or if $\beta=q+1$ and $M_{3}<M_{2}$ we deduce from (3.40), as in the proof of Theorem 3.1, the uniform estimate on the energy,

$$
\begin{gathered}
\underset{\tau \geq 0}{\sup \operatorname{ess}} \int_{\mathbf{R}^{N}} f(u(\tau, x)) d x+\int_{0}^{+\infty} \int_{\mathbf{R}^{N}} \mathcal{A}(\tau, x, u, D u) \cdot D u d \tau d x \\
\leq K \int_{\mathbf{R}^{N}} f\left(u_{0}(x)\right) d x,
\end{gathered}
$$

where $K$ is a structural constant. If we fix $y$ outside $B_{R+1}(0)$ and set $\rho_{0}=|y|-R$, we get as in Step 1

$$
\{x: u(t, x)=0\} \supset B_{\rho_{1}}(y) \quad \forall t \in\left[0, T_{\rho}\right] \cap[0, T],
$$

with $\rho_{1}^{\nu}(t)=\rho_{0}^{\nu}-M \max \left(t^{\lambda}, t^{\lambda} T^{1 / q-\lambda}\right) \rho_{0}^{\nu-1}$ with $M$ depending only on the structural constants and $\left\|u_{0}\right\|_{L^{(m+1) / m}\left(\mathbf{R}^{N}\right)}$ and $T$ is arbitrary. From the mean value theorem

$$
\rho_{0}^{\nu}-\rho_{1}^{\nu}(t)=\nu \tilde{\rho}^{\nu-1}\left(\rho_{0}-\rho_{1}(t)\right)=M \max \left(t^{\lambda}, T^{1 / q-\lambda} t^{\lambda}\right) \rho_{0}^{\nu-1},
$$

where $\tilde{\rho} \in\left(\rho_{1}(t), \rho_{0}\right)$, so

$$
\rho_{0}-\rho_{1}(t)=\frac{M}{\nu} \max \left(t^{\lambda}, T^{1 / q-\lambda} t^{\lambda}\right)\left(\frac{\rho_{0}}{\tilde{\rho}}\right)^{\nu-1} .
$$

Moreover $\operatorname{supp} u(t, \cdot) \in B_{R+\rho_{0}-\rho_{1}(t)}(0)$. As

$$
1-\left(\frac{\rho_{1}}{\rho_{0}}\right)^{\nu}=\frac{M}{\nu \rho_{0}} \max \left(t^{\lambda}, T^{1 / q-\lambda} t^{\lambda}\right)
$$

we deduce $\lim _{|y| \rightarrow+\infty}\left(\rho_{0} / \rho_{1}\right)=\lim _{|y| \rightarrow+\infty}\left(\rho_{0} / \tilde{\rho}\right)=1$ and

$$
\operatorname{supp} u(t, \cdot) \subset\left\{x:|x| \geq R+\frac{M}{\nu} \max \left(t^{\lambda}, T^{1 / q-\lambda} t^{\lambda}\right)\right\}
$$

for $t \leq \lim _{\rho \rightarrow+\infty} T_{\rho}=+\infty$. In particular we can take $t=T$ and we get (3.38).

REMARK 3.3. When $B \neq 0$ and $\beta<q+1$ we do not have the estimate (3.41) so (3.38) is only valid for $t \leq T<T^{*}$ with a constant $M$ depending on $T$. Moreover we do not know whether the relation (3.37) (which says that the energy of a solution is locally uniform in $\mathbf{R}^{N}$ ) is necessary in order to get the finite speed of propagation of the support of $u$.

REMARK 3.4. When $A(\cdot, \cdot, u, D u)=|D u|^{q-1} D u$ and $B=0$, then the phenomenon described in Corollary 3.1 is already known (see $[\mathbf{5}, \mathbf{1 4}, \mathbf{1 6}, \mathbf{2 1}, \mathbf{2 5}]$ and [17] for first order quasilinear equations). On the other hand if $q m \leq 1$, then the speed of propagation of the support of $u(t, \cdot)$ is infinite (see $[5,16,19$ and 32]).

In the next theorem we obtain the localization of the support of $u(t, \cdot)$ independently of $t$. Such a result is already known for specific first and second order quasilinear variational inequalities under some assumptions of monotonicity (see $[16,17$ and 20]).

THEOREM 3.2. Assume that $M_{2}>0, M_{4}>0, \sigma \geq 0, q>0, m>0, c>0$, $0 \leq \beta \leq q+1, \alpha=\sigma-\beta(\sigma+1) /(q+1)$ and $M_{3}<M_{4}\left(\right.$ resp. $\left.M_{3}<M_{2}\right)$ if $\beta=0$ (resp. $\beta=q+1)$ or

$$
M_{3}<\left(M_{4} \frac{q+1}{q+1-\beta}\right)^{(q+1-\beta) /(q+1)}\left(M_{2} \frac{q+1}{\beta}\right)^{\beta /(q+1)}
$$


if $0<\beta<q+1$; we assume moreover that $\max (\sigma, 1 / m)<q$. If $u$ is a weak solution of $(\mathrm{PE})$ in $\mathbf{R}^{+} \times \Omega$ with an initial data $u_{0}$ vanishing in $B_{\rho_{0}}\left(x_{0}\right), x_{0} \in \Omega$, $\rho_{0}<\operatorname{dist}\left(x_{0}, \partial \Omega\right)$, and if $u$ has a finite energy in $\mathbf{R}^{+} \times B_{\rho_{0}}\left(x_{0}\right)$ that is $j(u) \in$ $L^{\infty}\left(\mathbf{R}^{+}, L^{\mathbf{1}}\left(B_{\rho_{0}}\left(x_{0}\right)\right)\right), u \in L^{\sigma+1}\left(\mathbf{R}^{+} \times B_{\rho_{0}}\left(x_{0}\right)\right)$ and $D u \in L^{q+1}\left(\mathbf{R}^{+} \times B_{\rho_{0}}\left(x_{0}\right)\right)$, then $u(t, x)=0$ for almost all $(t, x)$ in $\mathbf{R}^{+} \times B_{\rho_{1}}\left(x_{0}\right)$, where

$$
\begin{aligned}
\rho_{1}^{\nu}=\rho_{0}^{\nu}-C \min _{(\tilde{\varepsilon}+1) /(q+1)<\tau \leq 1} & \left\{\frac{E^{\gamma}\left(\rho_{0}\right)}{\tau(q+1)-\tilde{\varepsilon}-1}\right. \\
& \left.\max \left(\left(b\left(\rho_{0}\right)+c\left(\rho_{0}\right)\right)^{\mu},\left(b\left(\rho_{0}\right)+c\left(\rho_{0}\right)\right)^{\eta}\right) \max \left(1, \rho_{0}^{\chi}\right)\right\},
\end{aligned}
$$

where $C$ depends on the $M_{i}, N, m, q, \sigma, c$ and

$$
\begin{gathered}
E(\rho)=\int_{0}^{+\infty} \int_{B_{\rho_{0}}\left(x_{0}\right)} A(\tau, x, u, D u) \cdot D u d x d \tau, \\
b(\rho)=\operatorname{supess}_{\tau \geq 0} \int_{B_{\rho}\left(x_{0}\right)} f(u(\tau, x)) d x, \ldots, \\
c(\rho)=\int_{0}^{+\infty} \int_{B_{\rho}\left(x_{0}\right)}|u(\tau, x)|^{\sigma+1} d x d \tau, \\
\gamma=\frac{(\varepsilon+1)(\tau(q+1)-\tilde{\varepsilon}-1)}{(\tilde{\varepsilon}+1)(N(q-\varepsilon)+(q+1)(\varepsilon+1))}, \\
\chi=\nu-1+\frac{((q+1)(m+1)+N m(q-\sigma))(N(q-\varepsilon)+\varepsilon+1)}{q(m+1)(N(q-\varepsilon)+(\varepsilon+1)(q+1))} \\
\mu=\frac{(m \sigma-1)(m+1)(N(q-\varepsilon)+(q+1)(\varepsilon+1))}{(\tilde{\varepsilon}+1)(N(q-\varepsilon)+(\varepsilon+1)(q+1))}, \ldots, \\
\eta=\mu+\frac{(\tilde{\varepsilon}-\varepsilon)(q+1)}{q(\varepsilon+1)(\tilde{\varepsilon}+1)}+\frac{(q-\tilde{\varepsilon})(N(q-\varepsilon)+\varepsilon+1)}{q(\tilde{\varepsilon}+1)(N(q-\varepsilon)+(q+1)(\varepsilon+1))}
\end{gathered}
$$

in which formulas we have set $\varepsilon=\min (\sigma, 1 / m)$ and $\tilde{\varepsilon}=\max (\sigma, 1 / m)$.

We first need the following $(N+1)$-dimensional trace-interpolation estimate. For the sake of simplicity let $Q_{\rho, t}=(0, t) \times B_{\rho}\left(x_{0}\right)$ and $\Sigma_{\rho, t}=(0, t) \times S_{\rho}\left(x_{0}\right)$, $(t, \rho) \in[0,+\infty] \times\left[0, \rho_{0}\right]$.

LEMMA 3.2. Assume that $u \in L^{\infty}\left(0, t ; L^{(m+1) / m}\left(B_{\rho}\left(x_{0}\right)\right)\right) \cap L^{\sigma+1}\left(Q_{\rho, t}\right)$ and $D u \in L^{q+1}\left(Q_{\rho, t}\right)$, (3.42) being satisfied. Then $u \in L^{q+1}\left(\Sigma_{\rho, t}\right)$ and there exists a nonnegative constant $C=C(N, q, \sigma, m)$ such that

$$
\begin{aligned}
\|u\|_{L^{q+1}\left(\Sigma_{\rho, t}\right) \leq} & C \max \left(1, \rho^{\lambda}\right)\left(\|D u\|_{L^{q+1}\left(Q_{\rho, t}\right)}+\rho^{\zeta}\left(\|u\|_{L^{\sigma+1}\left(Q_{\rho, t}\right)}\right.\right. \\
& \left.\left.+\sup _{0 \leq \tau \leq t} \operatorname{ess}\|u(\tau, \cdot)\|_{L^{(m+1) / m}\left(B_{\rho}\left(x_{0}\right)\right)}\right)\right)^{\theta} \\
& \times\left(\|u\|_{L^{\sigma+1}\left(Q_{\rho, t}\right)}+\sup _{0 \leq \tau \leq t} \operatorname{ess}\|u(\tau, \cdot)\|_{L^{(m+1) / m}\left(B_{\rho}\left(x_{0}\right)\right)}\right)^{1-\theta}
\end{aligned}
$$


where

$$
\begin{gathered}
\zeta=-1-\frac{N m(q-\sigma)}{(q+1)(m+1)}, \quad \theta=\frac{N(q-\varepsilon)+\varepsilon+1}{N(q-\varepsilon)+(\varepsilon+1)(q+1)} \\
\lambda=\left(\frac{(m \varepsilon-1)(q-\sigma)}{(m \sigma-1)(m+1)}+\frac{\sigma-\varepsilon}{m \sigma-1}\right) \frac{N q|1-m \sigma|}{(q+1)(N(q-\varepsilon)+(q+1)(\varepsilon+1))}
\end{gathered}
$$

where $\varepsilon=\min (\sigma, 1 / m)$ (notice that $\lambda=0$ if $\sigma=1 / m)$.

PROOF. First case: $\sigma m \leq 1$. Applying Corollary 2.1 yields

$$
\begin{aligned}
& \|u(\tau, \cdot)\|_{L^{q+1}\left(S_{\rho}\left(x_{0}\right)\right)}^{q+1} \\
& \quad \leq C^{q+1}\left(\|D u\|_{L^{q+1}\left(B_{\rho}\left(x_{0}\right)\right)}+\rho^{\delta}\|u(\tau, \cdot)\|_{L^{\sigma+1}\left(B_{\rho}\left(x_{0}\right)\right)}\right)^{\theta(q+1)} \\
& \cdots\|u(\tau, \cdot)\|_{L^{\sigma+1}\left(B_{\rho}\left(x_{0}\right)\right)}^{(1-\theta)}
\end{aligned}
$$

with

$$
\delta=\delta(\sigma)=-\frac{N(q-\sigma)+(q+1)(\sigma+1)}{(q+1)(\sigma+1)}, \quad \theta=\theta(\sigma)=\frac{N(q-1)+\sigma+1}{N(q-\sigma)+(q+1)(\sigma+1)} .
$$

From Hölder's inequality we have

$$
\begin{aligned}
& \int_{0}^{t} \int_{S_{\rho}\left(x_{0}\right)}|u|^{q+1} d s d \tau \\
& \leq(2 C)^{q+1}\left(\int_{0}^{t} \int_{B_{\rho}\left(x_{0}\right)}|D u|^{q+1} d x d \tau+\rho^{\delta(q+1)} \int_{0}^{t}\|u\|_{L^{\sigma+1}\left(B_{\rho}\left(x_{0}\right)\right)}^{q+1} d \tau\right)^{\theta} \\
& \cdots\left(\int_{0}^{t}\|u\|_{L^{\sigma+1}\left(B_{\rho}\left(x_{0}\right)\right)}^{q+1} d \tau\right)^{1-\theta}
\end{aligned}
$$

and taking the $(q+1)$ th root

$$
\begin{gathered}
\|u\|_{L^{q+1}\left(\Sigma_{\rho, t}\right)} \leq 2 C\left(\|D u\|_{L^{q+1}\left(Q_{\rho, t}\right)}+\rho^{\delta}\left(\int_{0}^{t}\|u\|_{L^{\sigma+1}\left(B_{\rho}\left(x_{0}\right)\right)}^{q+1} d \tau\right)^{1 /(q+1)}\right)^{\theta} \\
\cdots\left(\int_{0}^{t}\|u\|_{L^{\sigma+1}\left(B_{\rho}\left(x_{0}\right)\right)}^{q+1} d \tau\right)^{(1-\theta) /(q+1)} .
\end{gathered}
$$

But on the other hand

$$
\left(\int_{0}^{t}\|u\|_{L^{\sigma+1}\left(B_{\rho}\left(x_{0}\right)\right)}^{q+1} d \tau\right)^{1 /(q+1)} \leq\|u\|_{L^{\infty}\left(0, t ; L^{\sigma+1}\left(B_{\rho}\left(x_{0}\right)\right)\right)}^{(q-\sigma) /(q+1)}\|u\|_{L^{\sigma+1}\left(Q_{\rho, t}\right)}^{(\sigma+1) /(q+1)}
$$

Moreover

$$
\|u\|_{L^{\infty}\left(0, t ; L^{\sigma+1}\left(B_{\rho}\left(x_{0}\right)\right)\right)} \leq\left(\alpha_{N} \rho^{N}\right)^{1 /(\sigma+1)-m /(m+1)}\|u\|_{L^{\infty}\left(0, t ; L^{(m+1) / m}\left(B_{\rho}\left(x_{0}\right)\right)\right)},
$$

where $\alpha_{N}$ is the volume of the unit ball in $\mathbf{R}^{N}$; from Young's inequality we get

$$
\begin{gathered}
\left(\int_{0}^{t}\|u\|_{L^{\sigma+1}\left(B_{\rho}\left(x_{0}\right)\right)}^{q+1} d \tau\right)^{1 /(q+1)} \leq\left(\alpha_{N} \rho^{N}\right)^{(q-\sigma)(1-m \sigma) /(q+1)(\sigma+1)(m+1)} \\
\times\left\{\|u\|_{L^{\infty}\left(0, t ; L^{(m+1) / m}\left(B_{\rho}\left(x_{0}\right)\right)\right)}+\|u\|_{L^{\sigma+1}\left(Q_{\rho, t}\right)}\right\} .
\end{gathered}
$$


Combining (3.54) and (3.55) we obtain (3.50) with

and

$$
\varsigma=\delta(\sigma)+\frac{N(q-\sigma)(1-m \sigma)}{(q+1)(m+1)(\sigma+1)}=-1-\frac{N m(q-\sigma)}{(q+1)(m+1)}
$$

$$
\lambda=\frac{(1-\theta(\sigma)) N(q-\sigma)(1-m \sigma)}{(\sigma+1)(q+1)(m+1)}=\frac{N q(q-\sigma)(1-m \sigma)}{(q+1)(m+1)(N(q-\sigma)+(q+1)(\sigma+1))} .
$$

Second case: $\sigma m>1$. We apply Corollary 2.1 with $\sigma$ replaced by $1 / m$ and we get (3.54) with $\sigma$ replaced by $1 / m$. By interpolation we have

$$
\begin{aligned}
& \left(\int_{0}^{t}\|u\|_{L^{(m+1) / m}\left(B_{\rho}\left(x_{0}\right)\right)}^{q+1} d \tau\right)^{1 /(q+1)} \\
& \quad \leq\|u\|_{L^{\infty}\left(0, t ; L^{(m+1) / m}\left(B_{\rho}\left(x_{0}\right)\right)\right)}^{(q-\sigma) /(q+1)}\|u\|_{L^{\sigma+1}\left(0, t ; L^{(m+1) / m}\left(B_{\rho}\left(x_{0}\right)\right)\right)}^{(\sigma+1) /(q+1)}
\end{aligned}
$$

Moreover

$$
\|u\|_{L^{\sigma+1}\left(0, t ; L^{(m+1) / m}\left(B_{\rho}\left(x_{0}\right)\right)\right)} \leq\left(\alpha_{N} \rho^{N}\right)^{m /(m+1)-1 /(\sigma+1)}\|u\|_{L^{\sigma+1}\left(Q_{\rho, t}\right)},
$$

and we get

$$
\begin{aligned}
& \left(\int_{0}^{t}\|u\|_{L^{(m+1) / m}\left(B_{\rho}\left(x_{0}\right)\right)}^{q+1} d \tau\right)^{1 /(q+1)} \\
& \leq\left(\alpha_{N} \rho^{N}\right)^{(m \sigma-1) /(m+1)(q+1)} \\
& \quad \times\left\{\|u\|_{L^{\infty}\left(0, t ; L^{(m+1) / m}\left(B_{\rho}\left(x_{0}\right)\right)\right)}+\|u\|_{L^{\sigma+1}\left(Q_{\rho, t}\right)}\right\}
\end{aligned}
$$

and finally we obtain (3.50) with

$$
\varsigma=\delta\left(\frac{1}{m}\right)+\frac{N(m \sigma-1)}{(m+1)(q+1)}=-1-\frac{N m(q-\sigma)}{(q+1)(m+1)}
$$

and

$$
\lambda=\frac{N(m \sigma-1)}{(m+1)(q+1)}\left(1-\theta\left(\frac{1}{m}\right)\right)=\frac{N q(m \sigma-1)}{(q+1)(N(m q-1)+(q+1)(m+1))} .
$$

Proof of TheOREM 3.2. As in Lemma 3.1, $A(\cdot, \cdot, u, D u) \cdot D u, B(\cdot, \cdot, u, D u) u$, $|u|^{\sigma+1}$ and $u|A(\cdot, \cdot, u, D u)|$ belong to $L^{1}\left(Q_{\rho, t}\right)$ and for almost all $\rho \in\left(0, \rho_{0}\right)$ and $t>0$ we have

$$
\begin{aligned}
\int_{B_{\rho}\left(x_{0}\right)} f(u(t, x)) & d x+\iint_{Q_{\rho, t}}\{A(\tau, x, u, D u) \cdot D u+B(\tau, x, u, D u) u\} d x d \tau \\
& +M_{4} \iint_{Q_{\rho, t}}|u|^{\sigma+1} d x d \tau \\
\leq & \int_{B_{\rho}\left(x_{0}\right)} f\left(u_{0}(x)\right) d x+\iint_{\Sigma_{\rho, t}} A(\tau, x, u, D u) \cdot \vec{\nu} u d s d \tau
\end{aligned}
$$

Moreover, as in the proof of Theorem 2.1, (3.42) implies that there exists $M_{5}>0$ such that

$$
\begin{gathered}
\iint_{Q_{\rho, t}}\left\{A(\tau, x, u, D u) \cdot D u+B(\tau, x, u, D u) u+M_{4}|u|^{\sigma+1}\right\} d x d \tau \\
\geq M_{5} \int_{Q_{\rho, t}} \int\left(A(\tau, x, u, D u) \cdot D u+|u|^{\sigma+1}\right) d x d \tau
\end{gathered}
$$


and from Hölder's inequality

$$
\begin{aligned}
& \iint_{\Sigma_{\rho, t}} A(\tau, x, u, D u) \cdot \vec{\nu} u d s d \tau \\
& \quad \leq M_{1}\left(\iint_{\Sigma_{\rho, t}}|D u|^{q+1} d s d \tau\right)^{q /(q+1)}\left(\iint_{\Sigma_{\rho, t}}|u|^{q+1} d s d \tau\right)^{1 /(q+1)} .
\end{aligned}
$$

As $u_{0}$ vanishes in $B_{\rho}\left(x_{0}\right)$, we deduce as in Theorems 2.1 and 3.1,

$$
\begin{aligned}
& M_{6}\left(E(t, \rho)+c(t, \rho)+\int_{B_{\rho}\left(x_{0}\right)}|u(t, x)|^{(m+1) / m} d x\right) \\
& \quad \leq M_{1} M_{2}^{-q /(q+1)}\left(\frac{\partial E}{\partial \rho}(t, \rho)\right)^{q /(q+1)}\|u\|_{L^{q+1}\left(\Sigma_{\rho, t}\right)}
\end{aligned}
$$

where $E(t, \rho)$ is defined in (3.4) and $c(t, \rho)=\iint_{Q_{\rho, t}}|u|^{\sigma+1} d x d \tau$. As the functions $E(t, \rho), c(t, \rho)$ and $\partial E(t, \rho) / \partial \rho$ are nondecreasing with $t,(3.58)$ remains valid if we replace $\int_{B_{\rho}\left(x_{0}\right)}|u(t, x)|^{(m+1) / m} d x$ by

$$
b(t, \rho)=\sup _{0 \leq \tau \leq t} \int_{B_{\rho}\left(x_{0}\right)}|u(\tau, x)|^{(m+1) / m} d x
$$

and $M_{1} M_{2}^{-q /(q+1)}$ by $2 M_{1} M_{2}^{-q /(q+1)}$. Using Lemma 3.2 we get

$$
\begin{aligned}
b(t, \rho)+c(t, \rho)+E(t, \rho) \leq M_{7} \max \left(1, \rho^{\lambda}\right)\left(\frac{\partial E}{\partial \rho}(t, \rho)\right)^{q /(q+1)} & \\
\times\left(E^{1 /(q+1)}(t, \rho)+\rho^{\varsigma}\left(b^{m /(m+1)}(\right.\right. & \left.\left.t, \rho)+c^{1 /(\sigma+1)}(t, \rho)\right)\right)^{\theta} \\
& \times\left(b^{m /(m+1)}(t, \rho)+c^{1 /(\sigma+1)}(t, \rho)\right)^{1-\theta},
\end{aligned}
$$

where $M_{7}$ depends on $M_{1}, M_{2}, M_{3}, M_{4}, N, q, \sigma, m, c$ and $\beta$. If we set $d(t, \rho)=$ $b(t, \rho)+c(t, \rho)$ and $d\left(\rho_{0}\right)=b\left(\rho_{0}\right)+c\left(\rho_{0}\right)$, we get

$$
\begin{aligned}
b^{m /(m+1)}(t, \rho) & +c^{1 /(\sigma+1)}(t, \rho) \leq d(t, \rho)^{m /(m+1)}+d(t, \rho)^{1 /(\sigma+1)} \\
& \leq 2 \max \left(d\left(\rho_{0}\right)^{m /(m+1)}, d\left(\rho_{0}\right)^{1 /(\sigma+1)}\right)\left(\frac{d(t, \rho)}{d\left(\rho_{0}\right)}\right)^{1 /(\tilde{\varepsilon}+1)}
\end{aligned}
$$

as $d(t, \rho) \leq d\left(\rho_{0}\right)$ and $1 /(\tilde{\varepsilon}+1)=\min (m /(m+1), 1 /(\sigma+1))$. Finally we obtain

$$
b^{m /(m+1)}(t, \rho)+c^{1 /(\sigma+1)}(t, \rho) \leq K_{0} d^{1 /(\tilde{\varepsilon}+1)}(t, \rho)
$$

with

$$
K_{0}=2 \max \left(d\left(\rho_{0}\right)^{1 /(\sigma+1)-1 /(\tilde{\varepsilon}+1)}, d\left(\rho_{0}\right)^{m /(m+1)-1 /(\tilde{\varepsilon}+1)}\right) .
$$

We deduce from (3.60) that

$$
\begin{aligned}
E^{1 /(q+1)}(t, \rho)\left(b^{m /(m+1)}(t, \rho)+c^{1 /(\sigma+1)}\right. & (t, \rho))^{(1-\theta) / \theta} \\
& +\rho^{\varsigma}\left(b^{m /(m+1)}(t, \rho)+c^{1 /(\sigma+1)}(t, \rho)\right)^{1 / \theta}
\end{aligned}
$$

is smaller than

$$
\max \left(K_{0}^{1 / \theta}, K_{0}^{(1-\theta) / \theta}\right)\left(E^{1 /(q+1)}(t, \rho) d^{(1-\theta) / \theta(\tilde{\varepsilon}+1)}(t, \rho)+\rho^{\varsigma} d^{1 / \theta(\tilde{\varepsilon}+1)}(t, \rho)\right) .
$$


As in Theorems 2.1 and 3.1, for any $\tau \in[0,1)$ we have

$$
\begin{aligned}
& E^{1 /(q+1)}(t, \rho) d^{(1-\theta) / \theta(\tilde{\varepsilon}+1)}(t, \rho)+\rho^{\varsigma} d^{1 / \theta(\tilde{\varepsilon}+1)}(t, \rho) \\
& \quad \leq 2 \rho^{\varsigma} K_{1}^{1 / \theta} \max \left(1, \rho_{0}^{-\varsigma}\right)(E(t, \rho)+d(t, \rho))^{1 /(q+1)+\tau(1-\theta) / \theta(\tilde{\varepsilon}+1)}
\end{aligned}
$$

where we have set

$$
K_{1}=\max \left(d\left(\infty, \rho_{0}\right)^{(1-\tau)(1-\theta) /(\tilde{\varepsilon}+1)}, d\left(\infty, \rho_{0}\right)^{(1-\tau(1-\theta)) /(\tilde{\varepsilon}+1)-\theta /(q+1)}\right) .
$$

Hence $E$ satisfies the differential inequality

$$
E^{1+(1-\theta) / q-\tau(1-\theta)(q+1) / q(\tilde{\varepsilon}+1)}(t, \rho) \leq K_{2} \rho^{s \theta(q+1) / q} \frac{\partial E}{\partial \rho}(t, \rho),
$$

with $K_{2}=\left(4 K_{1} M_{7} \max \left(K_{0}, K_{0}^{1-\theta}\right) \max \left(1, \rho_{0}^{\lambda-\varsigma^{\theta}}\right)\right)^{(q+1) / q}$. Integrating (3.63) between $\rho_{1}$ and $\rho_{0}$ yields if $\tau>(\tilde{\varepsilon}+1) /(q+1)$,

$$
\begin{gathered}
\frac{K_{2} q(\tilde{\varepsilon}+1)}{(1-\theta)(\tau(q+1)-\tilde{\varepsilon}-1)}\left\{E^{(1-\theta)(\tau(q+1)-\tilde{\varepsilon}-1) / q(\tilde{\varepsilon}+1)}\left(t, \rho_{0}\right)\right. \\
\left.-E^{(1-\theta)(\tau(q+1)-\tilde{\varepsilon}-1) / q(\tilde{\varepsilon}+1)}\left(t, \rho_{1}\right)\right\} \\
\geq \frac{q}{q-\varsigma \theta(q+1)}\left(\rho_{0}^{1-\varsigma \theta(1+1 / q)}-\rho_{1}^{1-\varsigma \theta(1+1 / q)}\right) .
\end{gathered}
$$

Hence if

(3.65)

$$
\begin{aligned}
\rho_{1}^{1-\varsigma \theta(1+1 / q)} \leq & \rho_{0}^{1-\varsigma \theta(1+1 / q)} \\
& -\frac{K_{2} q(q-\varsigma \theta(q+1))}{(1-\theta)(\tau(q+1)-\tilde{\varepsilon}-1)} E^{(1-\theta)(\tau(q+1)-\tilde{\varepsilon}-1) / q(\tilde{\varepsilon}+1)}\left(t, \rho_{0}\right)
\end{aligned}
$$

we have $E\left(t, \rho_{1}\right)=0$. As $E\left(t, \rho_{0}\right) \leq E\left(\rho_{0}\right)$ we deduce that $E\left(t, \rho_{1}\right)=0$ for any $t \geq 0$ if $\rho_{1}$ satisfies (3.65) with $E\left(t, \rho_{0}\right)$ replaced by $E\left(\rho_{0}\right)$ and from (3.63) $u$ vanishes in $B_{\rho_{1}}\left(x_{0}\right) \times[0,+\infty)$. If we compute the exponents we get

$$
\begin{gathered}
1-\varsigma^{\theta}\left(1+\frac{1}{q}\right)=1+\left(1+\frac{m+1+N m(q-\sigma)}{q(m+1)}\right)\left(\frac{N(q-\varepsilon)+\varepsilon+1}{N(q-\varepsilon)+(\varepsilon+1)(q+1)}\right)=\nu \\
\frac{(1-\theta)(\tau(q+1)-\tilde{\varepsilon}-1)}{q(\tilde{\varepsilon}+1)}=\frac{(\varepsilon+1)(\tau(q+1)-\tilde{\varepsilon}-1)}{(\tilde{\varepsilon}+1)(N(q-\varepsilon)+(q+1)(\varepsilon+1))}=\gamma
\end{gathered}
$$

$(\lambda-\varsigma \theta)\left(1+\frac{1}{q}\right)$

$$
\begin{aligned}
=\left(1+\frac{1}{q}\right) & \left\{\left(\frac{(m \varepsilon-1)(q-\sigma)}{(m \sigma-1)(m+1)}+\frac{\sigma-\varepsilon}{m \sigma-1}\right)\right. \\
& \times \frac{N,|1-m \sigma|}{(q+1)(N(q-\varepsilon)+(q+1)(\varepsilon+1))} \\
& \left.+\frac{((m+1)(q+1)+N m(q-\sigma))(N(q-\varepsilon)+\varepsilon+1)}{(m+1)(q+1)(N(q-\varepsilon)+(\varepsilon+1)(q+1))}\right\}=\lambda .
\end{aligned}
$$

As

$$
\max \left(K_{0}, K_{0}^{1-\theta}\right)=\max \left(1, d^{(\tilde{\varepsilon}-\varepsilon) /(\varepsilon+1)(\tilde{\varepsilon}+1)}\left(\infty, \rho_{0}\right)\right)
$$


and

$$
\begin{aligned}
& K_{1} \max \left(K_{0}, K_{0}^{1-\theta}\right) \\
& \quad=\max \left(d^{(1-\tau)(1-\theta) /(\tilde{\varepsilon}+1)}\left(\infty, \rho_{0}\right), d^{1 /(\varepsilon+1)-\tau(1-\theta) /(\tilde{\varepsilon}+1)-\theta /(q+1)}\left(\infty, \rho_{0}\right)\right)
\end{aligned}
$$

and we get (3.43).

REMARK 3.5. If the energy of $u$ in $\mathbf{R}^{+} \times B_{\rho_{0}}\left(x_{0}\right)$ is not too large, then $\rho_{1}>0$ and there truly exists a cylinder $\mathbf{R}^{+} \times B_{\rho_{1}}\left(x_{0}\right)$ where $u$ is a.e. zero. On the opposite, if $u$ has not a finite energy in $\mathbf{R}^{+} \times B_{\rho_{0}}\left(x_{0}\right)$ we just obtain a finite speed of propagation for the nonvanishing set of $u$ in $\mathbf{R}^{+} \times B_{\rho_{0}}\left(x_{0}\right)$. Moreover if we know a priori that $u$ is bounded in $\mathbf{R}^{+} \times B_{\rho_{0}}\left(x_{0}\right)$, the hypucheses on $\alpha$ and $\beta$ can be relaxed as in Remark 3.2.

COROllary 3.2. Assume that $u$ is a weak solution of (PE) in $\mathbf{R}^{+} \times \mathbf{R}^{N}$ satisfying (3.37) such that $j(u) \in C^{0}\left(\mathbf{R}^{+} ; L_{\text {loc }}^{1}\left(\mathbf{R}^{N}\right)\right)$ and that $M_{2}>0, M_{4}>0$, $\sigma \geq 0, q>0, m>0, c>0,0 \leq \beta \leq q+1, \alpha=\sigma-\beta(\sigma+1) /(q+1)$ and $M_{3}<M_{4}$ (resp. $M_{3}<M_{2}$ ) if $\beta=0$ (resp. $\beta=q+1$ ) or (3.42) if $0<\beta<q+1$, and assume also that $\max (\sigma, 1 / m)<q$. If the initial data $u_{0}$ of $u$ vanishes outside $B_{R}(0)$, then there exists $R_{1} \geq R_{0}$ depending on the structural constants and $\left\|u_{0}\right\|_{L^{(m+1) / m}\left(\mathbf{R}^{N}\right)}$ such that for any $t \geq 0 u(t, \cdot)$ vanishes a.e. outside $B_{R_{1}}(0)$.

PROOF. As in Corollary 3.1 we first notice that the support of $u$ has a finite speed of propagation. If we apply Lemma 3.1 in $(0, t) \times \beta_{\rho}(0)$ we get $(3.57)$ and if $\rho$ goes to $+\infty$ we obtain

$$
\begin{array}{rl}
\int_{\mathbf{R}^{N}} & f(u(t, x)) d x \\
& +\int_{0}^{t} \int_{\mathbf{R}^{N}}\{\mathcal{A}(\tau, x, u, D u) \cdot D u+B(\tau, x, u, D u) u+C(t, x, u) u\} d x d \tau \\
\leq & \int_{\mathbf{R}^{N}} f\left(u_{0}(x)\right) d x,
\end{array}
$$

which implies (with (3.42)) the energy estimate

$$
\begin{aligned}
\underset{\tau \geq 0}{\sup \operatorname{ess}} \int_{\mathbf{R}^{N}} f(u(\tau, x)) d x & +\int_{0}^{+\infty} \int_{\mathbf{R}^{N}}\left\{A(\tau, x, u, D u) \cdot D u+|u|^{\sigma+1}\right\} d x d \tau \\
& \leq C \int_{\mathbf{R}^{N}} f\left(u_{0}(x)\right) d x
\end{aligned}
$$

where $C$ depends on the structural constants. We now fix $x_{0}$ outside $B_{R+1}(0), \rho_{0}=$ $\left|x_{0}\right|-R$ and apply Theorem 3.2 in $B_{\rho_{0}}\left(x_{0}\right)$ : there exists a constant $K$ depending on $C$ and $\left\|u_{0}\right\|_{L^{(m+1) / m}\left(\mathbf{R}^{N}\right)}$ such that $u$ is zero a.e. in $\mathbf{R}^{+} \times B_{\rho_{1}}\left(x_{0}\right)$, where

$$
\rho_{1}^{\nu}=\rho_{0}^{\nu}-K \rho_{0}^{\chi} \text {. }
$$

But

$$
\nu-\chi=1-\frac{N|1-m \sigma|((m \varepsilon-1)(q-\sigma)+(\sigma-\varepsilon)(m+1))}{(m \sigma-1)(m+1)(N(q-\varepsilon)+(q+1)(\varepsilon+1))}
$$

If we compute we get

$$
\nu-\chi= \begin{cases}\frac{(\sigma+1)(m N(q-\sigma)+(q+1)(m+1))}{(m+1)(N(q-\sigma)+(q+1)(\sigma+1))} & \text { if } \sigma \leq \frac{1}{m} \\ \frac{N m(q-\sigma)+(q+1)(m+1)}{N(m q-1)+(q+1)(m+1)} & \text { if } \sigma>\frac{1}{m} .\end{cases}
$$


So in both cases $\nu-\chi>0$ and $\rho_{1} \geq 0$ as soon as $\rho_{0} \geq K^{1 /(\nu-\chi)}$. Hence $u(t, \cdot)$ vanishes a.e. outside $B_{R+K^{1 /(\nu-x)}}(0)$.

REMARK 3.6. As in the elliptic case we can relax the hypotheses of continuity on $r \mapsto C(\tau, x, r)$ in order to treat some variational inequalities $(\sigma=0)$. Our results are also valid when there is a unilateral constraint on $u$ such as

$$
\left\{\begin{array}{l}
u \geq 0 \text { in } \Omega \\
\frac{\partial}{\partial t} \psi(u)-\operatorname{div} \mathcal{A}(\tau, x, u, D u)+B(\tau, x, u, D u) \geq f
\end{array}\right.
$$

in the following weak sense:

$$
\begin{gathered}
\int_{0}^{+\infty} \int_{\Omega}\left\{A(s, x, u, D u) \cdot D_{\varsigma}+B(s, x, u, D u)_{\zeta}-\psi(u(s, x)) \frac{\partial \zeta}{\partial t}\right\} d x d s \\
\geq \int_{\Omega} \psi\left(u_{0}(x)\right) \varsigma(0, x) d x+\int_{0}^{\infty} \int_{\Omega} f_{\zeta} d x d s
\end{gathered}
$$

for any $\varsigma \in C_{0}^{\infty}\left(\mathbf{R}^{+} \times \Omega\right), \varsigma \geq 0$. In order to obtain the results of Theorem 3.1 we just have to suppose $f \leq 0$ a.e. and the results of Theorem $3.2 f \leq-\varepsilon$ a.e., where $\varepsilon$ is a fixed positive constant, and $M_{3}$ small enough (see [13] for results on parabolic variational inequalities involving the maximum principle).

4. The interpolation-trace lemma. For the sake of simplicity we restrict ourselves to $v \in C^{1}(\bar{G})$ since $C^{1}(\bar{G})$ is dense in $W^{1, q+1}(G)$. The proof of Lemma 2.2 is divided onto four steps (see [9, Appendix] for a similar result).

First step. From a result of $\left[\mathbf{2 6}\right.$, p. 45], for any $\varepsilon>0$ there exists $C_{\varepsilon}>0$ such that for any $v \in C^{1}(\bar{G})$ the following holds:

$$
\|v\|_{L^{q+1}(G)} \leq \varepsilon\|D v\|_{L^{q+1}(G)}+C_{\varepsilon}\|v\|_{L^{\sigma+1}(G)} .
$$

If we set $C_{2}=\max \left(1+\varepsilon, C_{\varepsilon}|G|^{1-1 /(\sigma+1)}\right)$ we get

$$
\|v\|_{W^{1, q+1}(G)} \leq C_{2}\left(\|D v\|_{L^{q+1}(G)}+\|v\|_{L^{\sigma+1}(G)}\right) .
$$

Second step. We start from the elementary trace result [1]: there exists $C_{3}>0$ such that for any $u \in C^{1}(\bar{G})$ we have

$$
\|u\|_{L^{1}(\partial G)} \leq C_{3}\|u\|_{W^{1,1}(G)}
$$

and for $q>0$ we apply (4.3) to $u=v|v|^{q}, v \in C^{1}(\bar{G})$, so

$$
\int_{\partial G}|v|^{q+1} d \sigma \leq C_{3}\left\{(q+1) \int_{G}|v|^{q}|D v| d x+\int_{G}|v|^{q+1} d x\right\} .
$$

Since

we get

$$
\int_{G}|v|^{q}|D v| d x \leq\|D v\|_{L^{q+1}(G)}\|v\|_{L^{q+1}(G)}^{q}
$$

$$
\int_{\partial G}|v|^{q+1} d \sigma \leq C_{3}\left\{(q+1)\|D v\|_{L^{q+1}(G)}\|v\|_{L^{q+1}(G)}^{q}+\|v\|_{L^{q+1}(G)}^{q+1}\right\}
$$

which implies

$$
\|v\|_{L^{q+1}(\partial G)} \leq\left((q+1) C_{3}\right)^{1 /(q+1)}\|v\|_{W^{1, q+1}(G)}^{1 /(q+1)}\|v\|_{L^{q+1}(G)}^{q /(q+1)} .
$$


Third step. Set $0 \leq \sigma \leq q<\infty$. We claim that there exists a constant $C_{4}>0$ such that for any $v \in C^{1}(\overline{\bar{G}})$ we have

$$
\|v\|_{L^{q+1}(G)} \leq C_{4}\|v\|_{W^{1, q+1}(G)}^{((q+1) \theta-1) / q}\|v\|_{L^{\sigma+1}(G)}^{(q+1)(1-\theta) / q} .
$$

Case 1. Assume $q+1<N$. From Sobolev's inequality we have $\|v\|_{L^{\tau}(G)} \leq$ $C\|v\|_{W^{1, q+1}(G)}$ with $1 / \tau=1 /(q+1)-1 / N$. Moreover

$$
\|v\|_{L^{q+1}(G)} \leq\|v\|_{L^{\tau}(G)}^{1-\lambda}\|v\|_{L^{\sigma+1}(G)}^{\lambda}
$$

where $1 /(q+1)=\lambda /(\sigma+1)+(1-\lambda) / \tau$, that is

$$
\lambda=(q+1)(\sigma+1) / N(q-\sigma)+(q+1)(\sigma+1) .
$$

Hence with Sobolev's inequality

$$
\|v\|_{L^{q+1}(G)} \leq C^{1-\lambda}\|v\|_{W^{1, q+1}(G)}^{1-\lambda}\|v\|_{L^{\sigma+1}(G)}^{\lambda}
$$

and

$$
1-\lambda=\frac{N(q-\sigma)}{N(q-\sigma)+(q+1)(\sigma+1)}=\frac{(q+1) \theta-1}{q}, \quad \lambda=\frac{(q+1)(1-\theta)}{q} .
$$

Case 2. Assume $q+1 \geq N \geq 1$. We set $\alpha=(N+1) / 2, \rho=2(q+1) /(N+1)$, $\beta=(\sigma+1)(N+1) / 2(q+1)$ and $\alpha^{*}=\alpha N /(N-\alpha)\left(\alpha^{*}=\infty\right.$ if $\left.N=1\right)$. From Hölder's interpolating inequality we have

$$
\|u\|_{L^{\alpha}(G)} \leq\|u\|_{L^{\alpha *}(G)}^{1-\lambda}\|u\|_{L^{\beta}(G)}^{\lambda},
$$

where $1 / \alpha=(1-\lambda) / \alpha^{*}+\lambda / \beta((4.8)$ is valid even if $0<\beta<1$ with a simple change of function). From Sobolev's inequality we get

$$
\|u\|_{L^{\alpha}(G)} \leq C_{5}\|u\|_{W^{1, \alpha}(G)}^{1-\lambda}\|u\|_{L^{\beta}(G)}^{\lambda} .
$$

Now we set $u=v|v|^{\rho-1}$ and we have

and

$$
\begin{gathered}
\|u\|_{L^{\alpha}(G)}=\|v\|_{L^{\alpha \rho}(G)}^{\rho}=\|v\|_{L^{q+1}(G)}^{\rho}, \\
\|u\|_{L^{\beta}(G)}=\|v\|_{L^{\beta \rho}(G)}^{\rho}=\|v\|_{L^{\sigma+1}(G)}^{\rho}, \\
\|u\|_{W^{1, \alpha}(G)}=\|v\|_{L^{q+1}(G)}^{\rho}+\left(\int_{G}\left(\rho|v|^{\rho-1}|D v|\right)^{\alpha} d x\right)^{1 / \alpha}
\end{gathered}
$$

$$
\int_{G}\left(|v|^{\rho-1}|D v|\right)^{\alpha} d x \leq\left(\int_{G}|v|^{\alpha \rho} d x\right)^{1-1 / \rho}\left(\int_{G}|D v|^{\alpha \rho} d x\right)^{1 / \rho}
$$

which yields $\|u\|_{W^{1, \alpha}(G)} \leq \rho\|v\|_{L^{q+1}(G)}^{\rho-1}\|v\|_{W^{1, q+1}(G)}$ and (4.9) becomes

$$
\|v\|_{L^{q+1}(G)}^{\rho} \leq C_{6} \rho^{1-\lambda}\|v\|_{L^{q+1}(G)}^{(\rho-1)(1-\lambda)}\|v\|_{W^{1, q+1}(G)}^{1-\lambda}\|v\|_{L^{\sigma+1}(G)}^{\lambda \rho} .
$$

If we compute the exponents we get

$$
\frac{1-\lambda}{\lambda \rho+1-\lambda}=\frac{N(q-\sigma)}{(q+1)(\sigma+1)+N(q-\sigma)}=\frac{(q+1) \theta-1}{q}
$$

and

$$
\frac{\lambda \rho}{\lambda \rho+1-\lambda}=\frac{(q+1)(\sigma+1)}{N(q-\sigma)+(q+1)(\sigma+1)}=\frac{(q+1)(1-\theta)}{q},
$$

which is (4.5). 
Fourth step. End of the proof. We use (4.4) and (4.5) and get

$$
\|v\|_{L^{q+1}(\partial G)} \leq C_{7}\|v\|_{W^{1, q+1}(G)}^{1 /(q+1)}\|v\|_{W^{1, q+1}(G)}^{(q \theta+\theta-1) /(q+1)}\|v\|_{L^{\sigma+1}(G)}^{1-\theta},
$$

where $\theta=N(q-\sigma)+\sigma+1 / N(q-\sigma)+(q+1)(\sigma+1)$; using (4.2) yields finally

$$
\|v\|_{L^{q+1}(\partial G)} \leq C\left(\|D v\|_{L^{q+1}(G)}+\|v\|_{L^{\sigma+1}(G)}\right)^{\theta}\|v\|_{L^{\sigma+1}(G)}^{1-\theta} .
$$

\section{REFERENCES}

1. R. A. Adams, Sobolev spaces, Academic Press, New York, 1975.

2. H. W. Alt and S. Luckhaus, Quasilinear elliptic-parabolic differential equations, Math. Z. 183 (1983), 311-341.

3. S. N. Antoncev, On the localization of solutions of nomlinear degenerate elliptic and parabolic equations, Soviet Math. Dokl. 24 (1981), 420-424.

4. $\mathrm{R}$. Aris, The mathematical theory of diffusion and reaction in permeable catalysts, Clarendon Press, Oxford, 1975.

5. C. Atkinson and J. E. Bouillet, Some qualitative properties of solutions of a generalized diffusion equation, Math. Proc. Cambridge Philos. Soc. 86 (1979), 495-510.

6. H. Attouch and A. Damlamian, Application des méthodes de convexité et monotonie à l'étude de certaines équations quasilinéaires, Proc. Roy. Soc. Edinburgh Sect. A 79 (1977), 107-129.

7. A. Bamberger, Etude d'une équation doublernent non linéaire, J. Funct. Anal. 24 (1977), 148-155.

8. Ph. Benilan, H. Brezis and M. G. Crandall, $A$ semilinear equation in $L^{1}\left(\mathbf{R}^{N}\right)$, Ann. Scuola Norm. Sup. Pisa Cl. Sci. (4) 2 (1975), 523-555.

9. M. F. Bidaut-Veron, Variational inequalities of onder $2 m$ in unbounded domains, Nonlinear Anal. 6 (1982), 253-269.

10. H. Brezis, Monotonicity methods in Hilbert spaces and some applications to nonlinear partial differential equations, Contributions to Nonlinear Functional Analysis (E. Zarantonello, ed.), Academic Press, New York, 1971.

11. _ Solutions of variational inequalities with compact support, Uspekhi Mat. Nauk 129 (1974), 103-108.

12. H. Brezis and F. Browder, Strongly nonlinear elliptic boundary value problems, Ann. Scuola Norm. Sup. Pisa Cl. Sci. (4)5 (1976), 587-603.

13. H. Brezis and A. Friedman, Estimates on the support of solutions of parabolic variational inequalities, Illinois J. Math. 20 (1976), 82-97.

14. J. I. Diaz, Solutions with compact support for some degenerate parabolic problems, Nonlinear Anal. 3 (1979), 831-847.

15. J. I. Diaz and J. Hernandez, Some results on the existence of free boundaries for parabolic reactiondiffusion systems, Trends in Theory and Practice of Nonlinear Differential Equations (V. Lakshmikantham, ed.), Dekker, New York, 1984.

16. J. I. Diaz and M. A. Herrero, Estimates on the support of the solutions of some nonlinear elliptic and parabolic problems, Proc. Roy. Soc. Edinburgh Sect. A 89 (1981), 249-258.

17. J. I. Diaz and L. Veron, Existence theory and qualitative properties of the solutions of some first order quasilinear variational inequalities, Indiana Univ. Math. J. 32 (1983), 319-361.

18. M. E. Gurtin and R. C. Mac Camy, On the diffusion of biological populations, Math. Biosci. 33 (1977), 35-49.

19. A. S. Kalashnikov, On equations of the nonstationary-filtration type in which the perturbation is propagated at infinite velocity, Vestnik Moskov. Univ. Ser. I Mat. Mekh. 6 (1972), 45-49.

20. Vychisl. Mat. i Mat. Fiz. 14 (1974), 891-905.

21. _ On a nonlinear equation appearing in the theory of non-stationary filtration, Trudy Sem. Petrovsk. 4 (1978), 137-146.

22. R. Kersner, The behaviour of temperature fronts in media with nonlinear thermal conductivity under absorption, Vestnik Moskov. Univ. Ser. I Mat. Mekh. 33 (1978), 44-51.

23. __ Filtration with absorption: necessary and sufficient conditions for the propagation of perturbations to have finite velocity (to appear). 
24. __ Nomlinear heat conduction with absorption: space localization and extinction in finite time (to appear).

25. B. F. Knerr, The porous medium equation in one dimension, Trans. Amer. Math. Soc. 234 (1977), 381-415.

26. O. A. Ladyzhenskaya and N. N. Ural'tseva, Linear and quasilinear elliptic equations, Academic Press, New York, 1968.

27. O. A. Ladyzhenskaya, V. A. Solonnikov and N. N. Ural'tseva, Linear and quasilinear equations of parabolic type, Amer. Math. Soc. Transl., vol. 23, 1968.

28. J. L. Lions, Quelques méthodes de résolutions des problèmes aux limites non linéaires, Dunod, Paris, 1969.

29. L. K. Martinson and K. B. Pavlov, The effect of magnetic plasticity in non-Newtonian fluids, Magnit. Gidrodinamika 3 (1969), 69-75.

30. Unsteady shear flows of a conducting fluid with a rheological power law, Magnit. Gidrodinamika 4 (1970), 50-58.

31. O. A. Oleinik, A. S. Kalashnikov and C. Yui Lin, The Cauchy problem and boundary problems for equations of the type of nonstationary filtration, Izv. Akad. Nauk SSSR Ser. Mat. 22 (1958), 667-704.

32. L. A. Peletier, A necessary and sufficient condition for the existence of an interface in flows through porous media, Arch. Rational Mech. Anal. 56 (1974), 183-190.

33. G. Stampacchia, Equations elliptiques du second ordre à coefficients discontinus, Presses de l'Univ. de Montréal, 1966.

34. L. Veron, Equations d'évolution semi-linéaires du second ondre dans $L^{1}$, Rev. Roumaine Math. Pures Appl. 27 (1982), 95-123.

35. ___ Effets régularisants de semi-groupes non linéaires dans des espaces de Banach, Ann. Fac. Sci. Toulouse Math. (5) 1 (1979), 171-200.

Departamento de ECuaciones Funcionales, FaCultad de Ciencias, UniversiDAD COMPLUTENSE, MADRID 28040, SPAin

DÉPARTEMENT de MAThÉmatiques, FACUlté des SCIENCES, PARC DE GRANDMONT, 37200 TOURS, FRANCE 